\title{
Unravelling Protein-Protein
} Interaction Networks Linked to Aliphatic and Indole Glucosinolate Biosynthetic Pathways in Arabidopsis

\author{
Sebastian J. Nintemann ${ }^{1}$, Daniel Vik ${ }^{1}$, Julia Svozil' ${ }^{2}$, Michael Bak ${ }^{1}$, Katja Baerenfaller ${ }^{2}$, \\ Meike Burow ${ }^{1}$ and Barbara A. Halkier ${ }^{1 *}$ \\ ${ }^{1}$ Department of Plant and Environmental Sciences, Faculty of Science, DynaMo Center, University of Copenhagen, \\ Frederiksberg, Denmark, ${ }^{2}$ Department of Biology, ETH Zurich, Zurich, Switzerland
}

OPEN ACCESS

Edited by:

Alex Jones,

University of Warwick,

United Kingdom

Reviewed by:

Roque Bru-Martinez,

University of Alicante, Spain

Tamara Gigolashvili,

University of Cologne, Germany

*Correspondence:

Barbara A. Halkier

bah@plen.ku.dk

Specialty section:

This article was submitted to

Plant Proteomics,

a section of the journal

Frontiers in Plant Science

Received: 19 September 2017 Accepted: 14 November 2017 Published: 29 November 2017

Citation:

Nintemann SJ, Vik D, Svozil J, Bak M, Baerenfaller K, Burow $M$ and Halkier BA (2017) Unravelling

Protein-Protein Interaction Networks Linked to Aliphatic and Indole Glucosinolate Biosynthetic Pathways

in Arabidopsis.

Front. Plant Sci. 8:2028. doi: 10.3389/fpls.2017.02028
Within the cell, biosynthetic pathways are embedded in protein-protein interaction networks. In Arabidopsis, the biosynthetic pathways of aliphatic and indole glucosinolate defense compounds are well-characterized. However, little is known about the spatial orchestration of these enzymes and their interplay with the cellular environment. To address these aspects, we applied two complementary, untargeted approaches-split-ubiquitin yeast 2-hybrid and co-immunoprecipitation screens-to identify proteins interacting with CYP83A1 and CYP83B1, two homologous enzymes specific for aliphatic and indole glucosinolate biosynthesis, respectively. Our analyses reveal distinct functional networks with substantial interconnection among the identified interactors for both pathway-specific markers, and add to our knowledge about how biochemical pathways are connected to cellular processes. Specifically, a group of protein interactors involved in cell death and the hypersensitive response provides a potential link between the glucosinolate defense compounds and defense against biotrophic pathogens, mediated by protein-protein interactions.

Keywords: Arabidopsis thaliana, protein-protein interactions, glucosinolates, regulatory networks, pathway organization

\section{INTRODUCTION}

The cell is a busy place where many biological processes are regulated by physical or functional protein-protein interaction networks. Within metabolism, many biosynthetic pathways are proposed to be assembled into multi-enzyme complexes called metabolons (Srere, 1985). Organization into metabolons provides several advantages such as increase in local concentrations of the enzymes and their substrates, efficient sequestration of reactive intermediates and improved channeling of intermediates to increase flux (Bassard et al., 2012; Laursen et al., 2015, 2016; Dastmalchi et al., 2016; French et al., 2016), and there is increasing evidence for the existence of pathway-specific metabolons (Bassard and Halkier, 2017). Within the crowded intracellular environment where proteins are constantly coming into physical contact, it is an open question how multi-enzyme pathways are orchestrated in response to developmental and exogenous cues. Are they self-assembled, or are scaffolding proteins and chaperones involved? Additionally, how do specific pathways couple to surrounding protein-protein interaction networks within the cell? 
The glucosinolate defense compounds are suitable as model metabolites for studying pathway orchestration, as they are present in the model plant Arabidopsis thaliana (hereafter Arabidopsis) that offers extensive 'omics databases, bioinformatics tools as well as large mutant collections. In Arabidopsis, the glucosinolates are present as methioninederived aliphatic and tryptophan-derived indole glucosinolates. All of the enzymes in the biosynthetic pathways are known (Sønderby et al., 2010). The respective amino acid is converted to a glucosinolate in seven consecutive enzymatic reactions constituting the core pathway. The first two steps are carried out by different endoplasmic reticulum (ER) membranebound cytochrome P450s, CYP79s and CYP83s, followed by five steps catalyzed by the cytoplasmic enzymes GSTs, GGP1, SUR1, UGT74s and SOTs (Figure S1). Some of these enzymes, e.g., the soluble SUR1 and GGP1, are shared between the two pathways, whereas others such as the CYP79s and CYP83s are specific for the respective class of glucosinolates. In addition to the core pathway, formation of aliphatic glucosinolates requires chain-elongation of the precursor methionine, which is a process catalyzed by plastid-localized enzymes.

The glucosinolate pathways are proposed to be organized in metabolons (Halkier and Gershenzon, 2006). An unstable pathway intermediate, $S$-alkyl thiohydroximate, produced by GGP1, will intramolecularly cyclize and hence result in abortion of the pathway, if the intermediate is not further metabolized immediately. This suggests a tight coupling between at least GGP1 and the following SUR1 (Mikkelsen et al., 2004). Furthermore, as cytochrome P450 enzymes are typically ER membrane-anchored, it has been speculated that the P450s can serve as nucleation points that recruit the soluble enzymes to the ER surface by protein-protein interactions (Jørgensen et al., 2005; Ralston and Yu, 2006). In addition, UGT74B1 enzyme kinetics support a channeling mechanism to achieve efficient reaction speed at physiological substrate concentration and to avoid product inhibition (Kopycki et al., 2013). Indeed, investigations of specific protein-protein interactions within the pathway have revealed interactions between the UGT74 and SOT enzymes, independently confirmed by yeast 2-hybrid, bimolecular fluorescence complementation (BiFC) and Förster resonance energy transfer (FRET) measurements (Andersen et al., 2016).

Besides the intra-pathway interactions discussed above, several proteins that are not involved in glucosinolate biosynthesis interact with glucosinolate biosynthetic enzymes. CYP83A1 - in aliphatic glucosinolate biosynthesis-has several reported interactors (Manzano et al., 2008; Lalonde et al., 2010; Weis et al., 2013; Jones et al., 2014). Its interaction with the BAX INHIBITOR 1 (BI1) protein, which has a function in programmed cell death and response to pathogen infection (Weis et al., 2013), has been further investigated, and it was speculated that this interaction facilitates the assembly or stabilization of a glucosinolate biosynthetic enzyme complex (Weis et al., 2014). CYP83B1-in tryptophan-derived glucosinolate biosynthesis-is reported to interact with a gibberellin receptor that binds to DELLA proteins (Griffiths et al., 2006; Middleton et al., 2012).
The role of this interaction is unknown, but it might play a role in balancing growth and defense responses. Collectively, these findings suggest that the enzymes in the glucosinolate pathways are subject to diverse protein-protein interactions that crucially contribute to their regulation and interconnection with signaling pathways and cellular metabolism.

Toward our goal to understand pathway orchestration and to link the glucosinolate pathways to their underlying proteinprotein interaction networks, we screened for protein interactors in two types of untargeted screens. As baits, we used the pathwayspecific CYP83A1 and CYP83B1 that catalyze steps in aliphatic or indole glucosinolate biosynthesis, respectively. These are the only enzymes that can be non-redundantly assigned to either the aliphatic or indole core pathway (Bak and Feyereisen, 2001; Hansen et al., 2001; Naur et al., 2003; Sønderby et al., 2010). To maximize coverage, we performed several split-ubiquitin-based yeast 2-hybrid screens (Stagljar et al., 1998; Möckli et al., 2007) as well as complementary co-immunoprecipitation (Co-IP) assays followed by tandem mass spectrometry (MS/MS)-based protein identification. Despite little overlap in detected protein-protein interactions between the conducted screens, the detected proteins overlap functionally. Network analysis revealed that the two glucosinolate biosynthetic pathways show differences in the way they interconnect with their cellular environment. As an example, we investigate a small family of hypersensitive response (HR)like lesion-inducing proteins that interact with glucosinolate biosynthetic enzymes and provide a possible link to innate immunity.

\section{RESULTS}

\section{Candidates Identified by Tissue-Specific Split-Ubiquitin Yeast 2-Hybrid Screens}

To identify the protein-protein interaction networks for the aliphatic and indole glucosinolate pathways, we conducted split-ubiquitin-based yeast 2-hybrid screens using CYP83A1 and CYP83B1 tagged with the C-terminal part of ubiquitin as baits. We constructed cDNA prey libraries from tissues with particularly high CYP83A1 and CYP83B1 transcript levels, respectively, to enrich for cDNAs of naturally co-occurring proteins. Based on CYP83 transcript levels available in the eFP browser of the BAR database (Winter et al., 2007), cDNA libraries were generated from node tissue (center of rosettes of 3-week-old plants, high in CYP83A1 transcripts) and hypocotyl tissue (from 10-day-old plants, high in CYP83B1 transcripts) of Arabidopsis ecotype Col-0. To investigate the importance of the tissue context, both libraries were screened with the CYP83A1 and CYP83B1 baits.

We analyzed plasmids from 16 (CYP83A1 with hypocotyl library), 144 (CYP83A1 with node library), 25 (CYP83B1 with hypocotyl library) and 56 (CYP83B1 with node library) yeast colonies by sequencing. Using the basic local alignment search tool (BLAST) of The Arabidopsis Information Resource (TAIR, www.arabidopsis.org), we identified a total of 72 unique inserts, most of which represent full length or nearly full length coding sequences. Duplicates and sequences unambiguously belonging 
to proteins of the photosynthetic and the ribosomal machinery were excluded from further analysis, leaving a curated list of 60 candidate interactors (Table 1).

Most sequences were found only once, with few sequences identified several times, and one coding sequence found in 93 out of the total 241 sequenced plasmids (Figure S2A). PANTHER Gene Ontology (GO)-Slim biological processes protein localization and organelle organization were overrepresented in the detected interactors of CYP83A1, while no over-representation was found for the interactors of CYP83B1.

Of the 60 identified proteins, 30 were exclusively interacting with CYP83A1, while 23 proteins were only identified in the CYP83B1 screens (Figure S2B). Seven were found in screens with both CYP83A1 and CYP83B1, including the candidate with the highest number of total occurrences in our screens, At5G43460, annotated as HR-like lesion-inducing protein-related. The choice of cDNA library had an impact on the total number of identified candidates in accordance with the determined library transformation titer (Table S2), with overall more sequences found employing the library constructed from node tissue and with a low overlap of interactors found in both libraries with each bait.

Because of the small overlap between interactors found with a single bait with different libraries and the relatively high number of candidates found with both baits, we investigated the specificity of the interactions for 13 of the proteins in targeted yeast 2-hybrid, three of which were identified using both CYP83 baits, while ten had been found exclusively with either CYP83A1 or CYP83B1 as bait. Their coding sequences were inserted into the prey vector and interaction with CYP83A1, CYP83B1 and the unrelated control bait protein Large T was assessed (Figure S3). We found that all the tested preys interacted with both CYP83A1 and CYP83B1, and that seven of them also interacted with LargeT.

\section{Candidate Interacting Proteins Identified by Co-IP}

To identify a broad array of relevant interactors, we additionally performed Co-IP experiments followed by MS/MS-based protein identification as a complementary, principally different approach to our yeast 2-hybrid screens. Transgenic Arabidopsis lines expressing either CYP83A1 or CYP83B1 fused to the mVenus fluorescent protein driven by the respective native promoter enabled us to use the protein tag as epitope for Co-IP. Subjecting protein extracts from 14-day-old seedlings to our analysis identified a total of 1840 proteins. As selection criteria, potential interactors of CYP83A1 and CYP83B1 had to be identified with at least 5 spectra and to have 5 -fold more spectral counts in the respective affinity enrichments compared to the wildtype control. We identified 38 and 40 candidates for CYP83A1 and CYP83B1, respectively, while four candidates were found with both bait proteins (Tables 2, 3). In our experiment, the CYP83A1-mVenus and CYP83B1-mVenus Co-IPs serve as control for each other, and the low overlap between detection of interactors excludes a frequent occurrence of proteins interacting with the fluorescent protein tag instead of the cytochrome P450 bait.
Among the proteins detected to interact with CYP83A1 was Glutamate-cysteine ligase (GSH1 or PAD2, At4G23100), an enzyme in glutathione (GSH) biosynthesis (Parisy et al., 2007). Glutathione serves as sulfur donor for glucosinolate biosynthesis and accordingly, a pad 2 mutant had $80 \%$ reduced GSH levels and was affected in the synthesis of both aliphatic and indole glucosinolates (Schlaeppi et al., 2008). Functional categorization using all proteins quantified in the Co-IP experiment with the results of our CYP83A1 Co-IP as reference list and without correction for multiple testing revealed an enrichment of porphyrin-containing compound and polysaccharide metabolic processes. The enriched GO biological processes resulting from an analogous analysis for CYP83B1 interactors included $R N A$ metabolic and catabolic processes.

\section{Yeast 2-Hybrid and Co-IP Identified Complementary Types of Proteins}

We observed almost no overlap between the candidate lists generated by Co-IP and by the yeast 2-hybrid screens. To complement our results, we integrated previously reported interactions from the BioGrid database (ChatrAryamontri et al., 2015) into our lists. Only two candidate proteins were present in more than one of these datasets, namely the membrane steroid-binding protein 1 (MSBP1, At5G52240), which was identified with the CYP83A1 bait in our yeast 2-hybrid screen and had previously been reported as an interactor of CYP83A1 via a protein-fragment complementation assay (Jones et al., 2014), and Acclimation of Photosynthesis to Environment (APE1, At5G38660), found in our screens as an interactor of CYP83B1 in yeast 2-hybrid and as an interactor of CYP83A1 in Co-IP (Figure S4).

Due to the complementary nature of the two methods, they are not expected to identify a large number of common candidates. To address this, we compared the physical-chemical properties of the identified proteins. The yeast 2-hybrid approach identified comparatively short proteins with an average length of 257.7 amino acids, and thereby shorter than the average length of 402.4 amino acids for all Arabidopsis proteins in TAIR10. With an average length of 486.7 amino acids for CYP83A1 interactors and of 556.83 for CYP81B1 interactors, the Co-IP-identified proteins had overall longer primary sequences (Figure 1A). Additionally, we compared the isoelectric points of the candidates identified by the two methods. Co-IP-identified proteins showed pI values between 12 and 4, with an average of 7.4 for CYP83A1 interactors and 8.1 for CYP83B1 interactors. The candidates identified by yeast 2-hybrid showed a tendency toward either higher or lower isoelectric points, with an average pI of 8.2 and $63 \%$ of them having a pI between 8 and 12 (Figure 1B). Under the cytosolic pH conditions of Arabidopsis (Shen et al., 2013), most yeast 2-hybrid candidates would thus be positively or negatively charged.

To compare the hydrophobicity of the candidate interactors detected with the two methods, we obtained the average hydropathy values (GRAVY scores) of the proteins as a measure for hydrophobicity (Kyte and Doolittle, 1982). It is evident 
TABLE 1 | Curated list of candidate interactors found in untargeted yeast 2-hybrid library screens.

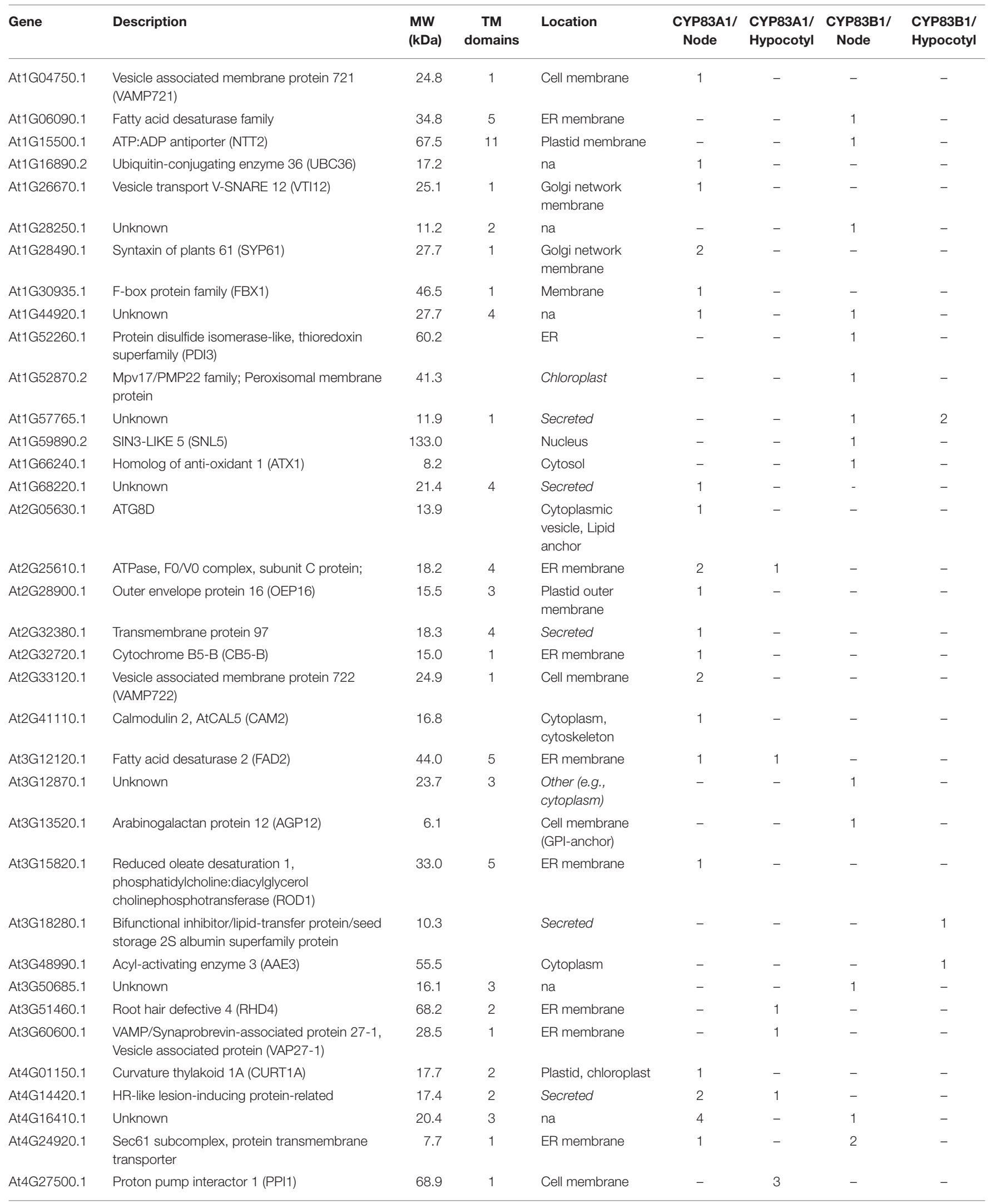


TABLE 1 | Continued

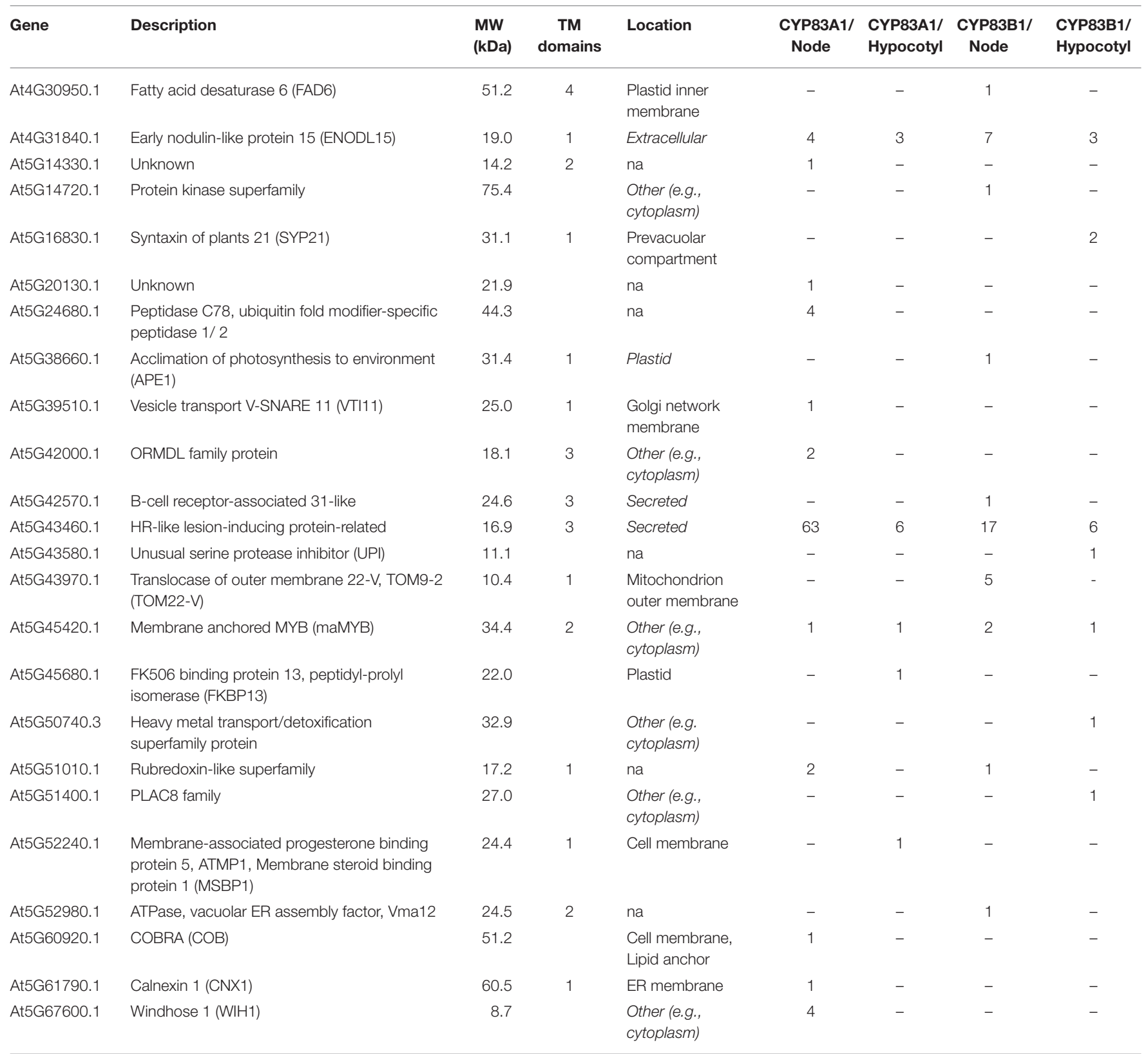

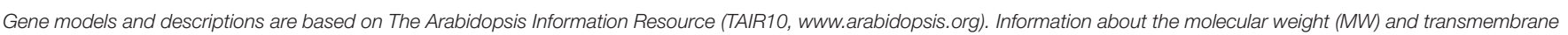

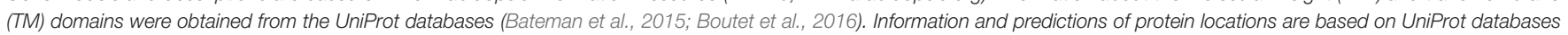

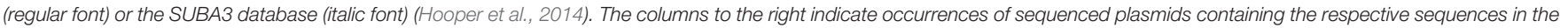
four screens with combinations of CYP83A1 or CYP83B1 as baits and libraries constructed from node and hypocotyl tissue. na, not available.

that the proteins identified in our yeast 2-hybrid screens showed a balanced distribution of positive and negative scores. In contrast, the pulldown candidates showed predominantly negative GRAVY scores, indicative of a bias toward overall hydrophilic proteins (Figure 1C). The number of proteins containing transmembrane domains in each list (Tables 1-3) supports this, as less than $10 \%$ of the candidates from the Co-IP experiments contain predicted transmembrane domains, while as much as around $70 \%$ of the candidates identified via yeast 2-hybrid contain at least one predicted transmembrane domain, underlining the clear difference in hydrophobicity.

To assess a possible bias against lowly expressed proteins, especially in the identified Co-IP candidates, we extracted the average expression values of the respective transcripts in vegetative rosettes from the eFP anatomy browser of the BAR database (Winter et al., 2007). As we had observed previously that absolute protein and transcript levels correlate positively, transcript expression values can be taken as a proxy for protein 
TABLE 2 | Curated list of candidate interactors found by Co-IP with CYP83A1-mVenus.

\begin{tabular}{|c|c|c|c|c|c|c|c|c|}
\hline Gene & Description & $\begin{array}{c}\text { MW } \\
\text { (kDa) }\end{array}$ & $\begin{array}{c}\text { TM } \\
\text { domains }\end{array}$ & Location & Exp1 & Exp2 & Exp3 & $\begin{array}{l}\text { Total } \\
\text { specs }\end{array}$ \\
\hline At1G11750 & CLP protease proteolytic subunit 6 (CLPP6) & 29.4 & & Plastid, stroma & 2 & 0 & 5 & 7 \\
\hline At1G14810 & Semialdehyde dehydrogenase family protein & 40.7 & & Plastid & 2 & 0 & 4 & 6 \\
\hline At1G20580 & Small nuclear ribonucleoprotein family protein (SMD3) & 14.2 & & Cytosol & 7 & 4 & 0 & 11 \\
\hline At1G54100 & Aldehyde dehydrogenase 7B4 (ALDH7B4) & 54.2 & & Cytosol & 1 & 0 & 5 & 6 \\
\hline At1G68830 & STT7 homolog (STN7) & 63.3 & & $\begin{array}{l}\text { Plastid, thylakoid } \\
\text { membrane }\end{array}$ & 0 & 0 & 5 & 5 \\
\hline At1G70730 & Phosphoglucomutase/phosphomannomutase family protein (PGM2) & 63.5 & & Cytoplasm & 3 & 0 & 4 & 7 \\
\hline At2G18960 & $\mathrm{H}(+)$-ATPase $1(\mathrm{HA} 1)$ & 104.2 & 10 & Cell membrane & 3 & 0 & 3 & 6 \\
\hline At2G40490 & Uroporphyrinogen decarboxylase (HEME2) & 43.6 & & Plastid & 2 & 0 & 3 & 5 \\
\hline At3G09200 & Ribosomal protein L10 family protein & 34.1 & & Cytosol & 6 & 0 & 6 & 12 \\
\hline At3G19170 & Presequence protease 1 (PREP1) & 121.0 & & $\begin{array}{l}\text { Plastid, stroma, } \\
\text { Mito., matrix }\end{array}$ & 6 & 1 & 7 & 14 \\
\hline At3G22142 & Encodes a Protease inhibitor/seed storage/LTP family protein & 147.2 & & Secreted & 8 & 0 & 0 & 8 \\
\hline At3G26070 & Plastid-lipid associated protein PAP / fibrillin family protein; & 27.2 & & Plastid & 0 & 0 & 6 & 6 \\
\hline At3G48000 & Aldehyde dehydrogenase 2 (ALDH2) & 58.6 & & $\begin{array}{l}\text { Mitochondrion, } \\
\text { matrix }\end{array}$ & 5 & 0 & 10 & 15 \\
\hline At3G48420 & Haloacid dehalogenase-like hydrolase superfamily protein & 34.2 & & Plastid & 5 & 0 & 1 & 6 \\
\hline At3G56240 & Copper chaperone $(\mathrm{CCH})$ & 13.0 & & Cytosol & 4 & 2 & 2 & 8 \\
\hline At3G57610 & Adenylosuccinate synthase (ADSS) & 53.0 & & Plastid & 3 & 0 & 3 & 6 \\
\hline At3G58730 & Vacuolar ATP synthase subunit D (VATPD) & 29.1 & & $\begin{array}{l}\text { Vacuole } \\
\text { membrane }\end{array}$ & 0 & 0 & 5 & 5 \\
\hline At3G61440 & Cysteine synthase C1 (CYSC1) & 39.9 & & Mitochondrion & 5 & 2 & 8 & 15 \\
\hline At4G13770 & Cytochrome P450, family 83, subfamily A, polypeptide 1 (CYP83A1) & 57.4 & 1 & ER membrane & 157 & 37 & 34 & 228 \\
\hline At4G15530 & Pyruvate orthophosphate dikinase (PPDK) & 105.1 & & Cytosol, Plastid & 0 & 0 & 7 & 7 \\
\hline At4G22240 & Plastid-lipid associated protein PAP / fibrillin family protein & 33.7 & & Plastid & 1 & 0 & 5 & 6 \\
\hline At4G23100 & Glutamate-cysteine ligase (GSH1) & 58.6 & & Plastid & 3 & 0 & 3 & 6 \\
\hline At4G23600 & Tyrosine transaminase family protein (CORI3) & 47.0 & & Cytosol & 0 & 2 & 11 & 13 \\
\hline At4G25080 & Magnesium-protoporphyrin IX methyltransferase (CHLM) & 33.8 & & $\begin{array}{l}\text { Plastid, peripheral } \\
\text { membrane }\end{array}$ & 1 & 0 & 5 & 6 \\
\hline At4G30270 & Xyloglucan endotransglucosylase/hydrolase 24 (XTH24) & 30.8 & & Secreted & 0 & 0 & 5 & 5 \\
\hline At4G33680 & Pyridoxal phosphate-dependent transferases superfam. prot. (AGD2) & 50.4 & & Plastid & 3 & 0 & 2 & 5 \\
\hline At5G11520 & Aspartate aminotransferase 3 (ASP3) & 49.0 & & Plastid & 1 & 0 & 4 & 5 \\
\hline At5G11670 & NADP-malic enzyme 2 (NADP-ME2) & 64.4 & & Cytoplasm & 5 & 0 & 1 & 6 \\
\hline At5G14200 & Isopropylmalate dehydrogenase 1 (IMD1) & 44.2 & & Plastid & 3 & 0 & 5 & 8 \\
\hline At5G14780 & Formate dehydrogenase (FDH) & 42.4 & & Mitochondrion & 6 & 1 & 7 & 14 \\
\hline At5G17710 & Co-chaperone GrpE fam. protein/embryo defective 1241 (EMB1241) & 35.5 & & $\begin{array}{l}\text { Mitochondrion, } \\
\text { matrix }\end{array}$ & 6 & 1 & 4 & 11 \\
\hline At5G19550 & Aspartate aminotransferase 2 (ASP2) & 44.3 & & Cytoplasm & 1 & 0 & 5 & 6 \\
\hline At5G38660 & Acclimation of photosynthesis to environment (APE1) & 31.4 & 1 & Plastid & 8 & 0 & 1 & 9 \\
\hline At5G42650 & Allene oxide synthase (AOS) & 58.2 & & Plastid & 6 & 0 & 15 & 21 \\
\hline At5G44130 & FASCICLIN-like arabinogalactan protein 13 precursor (FLA13) & 26.2 & & $\begin{array}{l}\text { Cell membrane, } \\
\text { Lipid anchor }\end{array}$ & 2 & 0 & 3 & 5 \\
\hline At5G49360 & Beta-xylosidase 1 (BXL1) & 83.5 & & Secreted & 0 & 0 & 11 & 11 \\
\hline At5G51820 & Phosphoglucomutase (PGM1) & 68.0 & & Plastid & 6 & 2 & 9 & 17 \\
\hline At5G60600 & 4-hydroxy-3-methylbut-2-enyl diphosphate synthase (HDS) & 82.3 & & Plastid, stroma & 1 & 0 & 10 & 11 \\
\hline
\end{tabular}

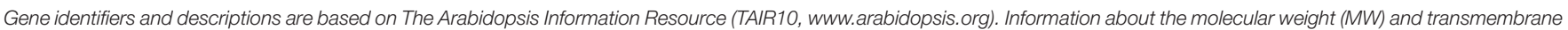

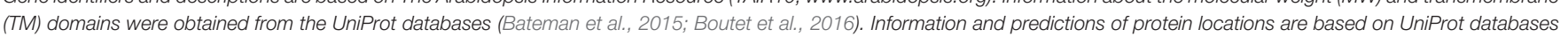

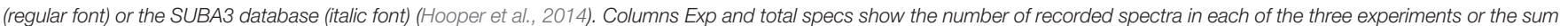
thereof, respectively.

abundance levels (Baerenfaller et al., 2008). The average log2transformed transcript abundance was 8.94 for the CYP81A1 interactors and 6.98 for the CYP81B1 interactors, respectively, while it was 7.4 for the interactors identified with yeast 2hybrid. Furthermore, the interactors identified with Co-IP did not show a correlation or a linear relationship between the 
TABLE 3 | Curated list of candidate interactors found by Co-IP with CYP83B1-mVenus.

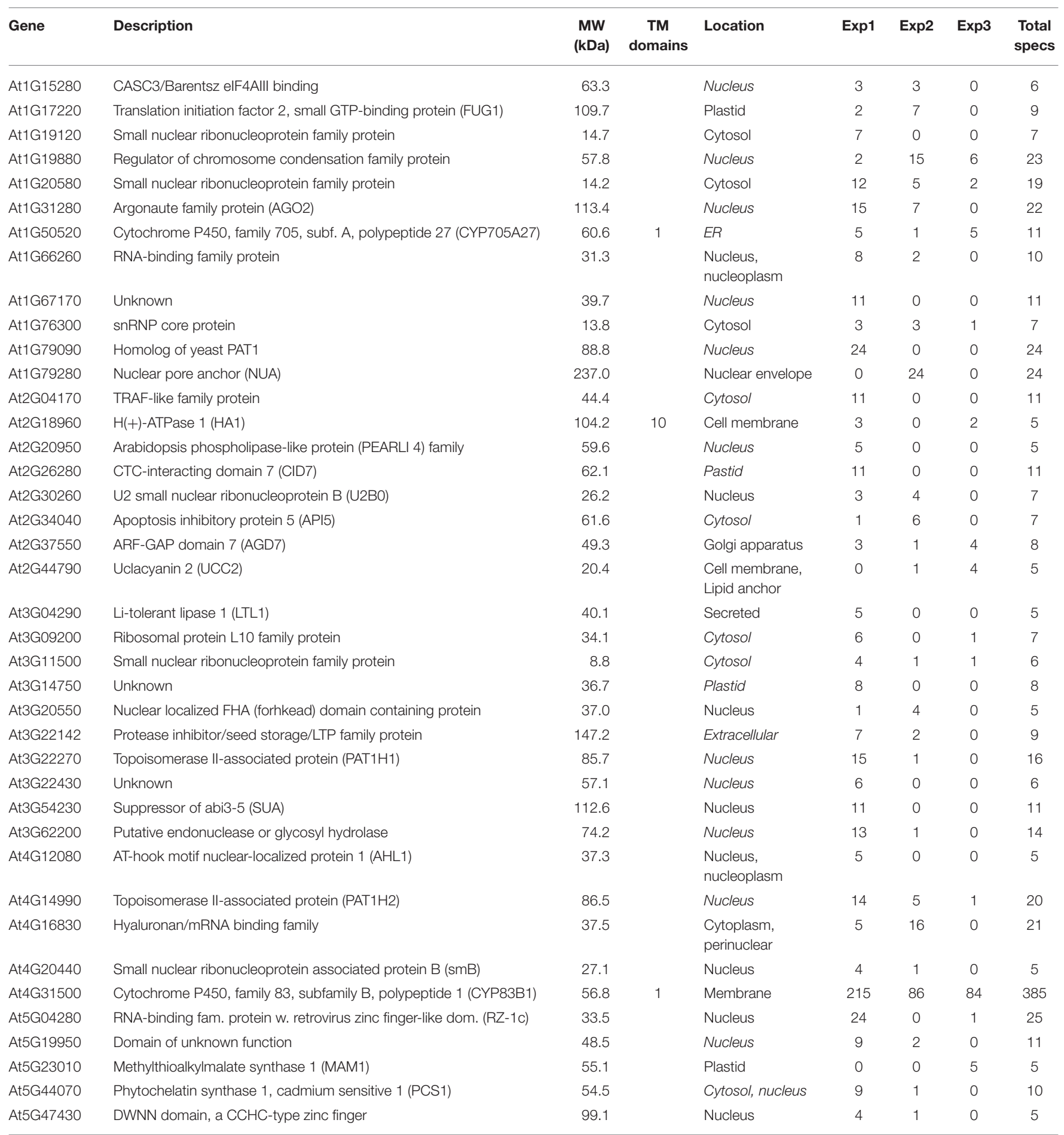

Gene identifiers and descriptions are based on The Arabidopsis Information Resource (TAIR10, www. arabidopsis.org). Information about the molecular weight (MW) and transmembrane (TM) domains were obtained from the UniProt databases (Bateman et al., 2015; Boutet et al., 2016). Information and predictions of protein locations are based on UniProt databases (regular font) or the SUBA3 database (italic font) (Hooper et al., 2014). Columns Exp and total specs show the number of recorded spectra in each of the three experiments or the sum thereof, respectively.

number of identified spectra and transcript abundance (Figure S5). The Co-IP therefore also identified candidates with very low transcript levels and, correspondingly, expected low protein levels. Thus, the bias for rather highly expressed proteins usually observed in high-throughput proteomics experiments was not detected here. 
A

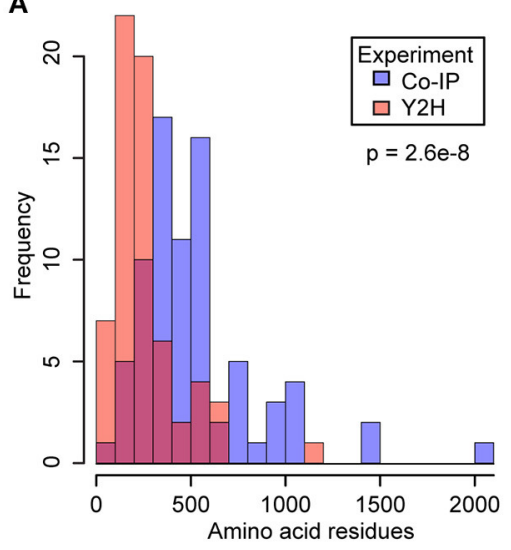

B

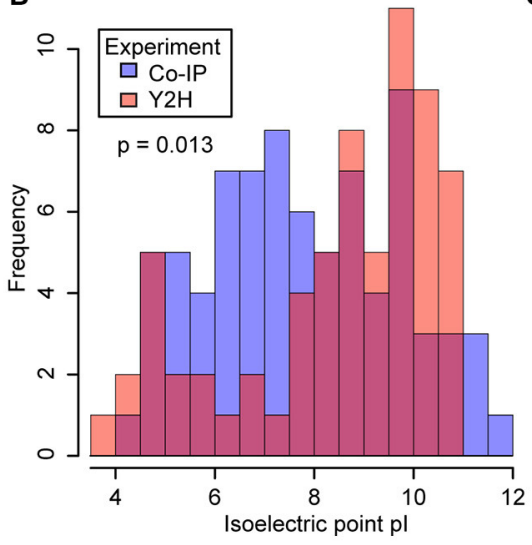

C

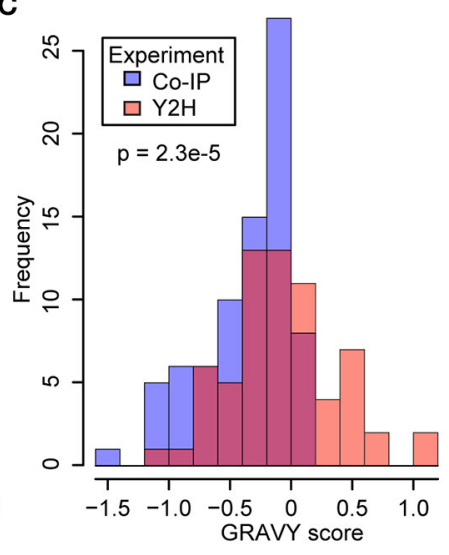

FIGURE 1 | Comparison of physical and chemical properties of proteins identified by yeast 2-hybrid (Y2H) and co-immunoprecipitation (Co-IP). Y2H, orange bars; Co-IP, blue bars; overlap, red. Protein lengths (A), isoelectric points (B) and hydrophobicity values as GRAVY scores (C) were obtained from the SUBA3 database (Hooper et al., 2014). The significance of the difference was tested by Welch two-sample t-tests (A,C) and a two-sample Kolmogorov-Smirnov test (B).

\section{Identified Candidates belong to Different Functional Networks}

To gain further insights into our datasets and to discover functional connections within the candidate interactor lists, we performed network analyses by submitting the candidate lists-together with the previously reported interactors from the BioGrid database-to network analysis using the STRING database (Szklarczyk et al., 2015). This analytical tool builds networks based on several reported factors, including physical interactions, but also other functional associations such as co-expression and participation in biological processes. The meta-analysis results in a score representing the connection between two proteins. In our analysis, we used a threshold score of 0.4 .

For interactors of CYP83A1, this resulted in a functional network with 81 nodes and 115 connections, significantly above the expected values for a random sample (48 connections expected, interaction enrichment $p$-value $=2.22 \mathrm{e}-16$; Figure 2A). Most proteins are included in two major subclusters. Cluster I consists mostly of candidate interactors detected in yeast 2-hybrid screens and is significantly enriched for GO categories organelle organization and vesicle-mediated transport. Interestingly, several interactors of the BI1 protein, which was previously reported to interact with CYP83A1, are present in cluster I, placing BI1 centrally in this cluster. Furthermore, the interaction of BI1 with CAM2 and HEME2 forms a direct link between clusters I and II. Cluster II contains proteins that were, apart from one exception, exclusively found in Co-IP, and GO categories generation of precursor metabolites and energy, as well as porphyrincontaining compound metabolic process are over-represented in this cluster.

The detected interactors of CYP83B1 form a network with 70 nodes and 45 connections (17 connections expected, interaction enrichment $p$-value $=2.04 \mathrm{e}-8$; Figure $2 B$ ). Here, interactors mostly detected by Co-IP constitute a cluster with proteins enriched in GO categories RNA catabolic process, RNA splicing, mRNA splicing, cellular component biogenesis and nitrogen compound metabolic process (cluster III). A second, independent cluster consisting of yeast 2-hybrid as well as two Co-IP candidates is not significantly enriched for functional categories. In contrast to the CYP83A1 network, the majority of CYP83B1 interactors could not be functionally linked to either of the clusters. While our network analysis underscores the individual strengths of the employed methods in detecting proteins of largely differing characteristics, functional connections could be drawn within but also between the datasets obtained by the complementary methods.

\section{Glucosinolate Profiles of Mutant Alleles of Candidate Interactors Suggest Biological Functions Impacting Glucosinolate Metabolism}

To assess the impact of the detected interactors on glucosinolate metabolism and thus assign an in planta function, we analyzed glucosinolate profiles of available homozygous SALK T-DNA insertion lines, targeting 34 of the 54 potential interactors identified with yeast 2-hybrid along with wildtype Arabidopsis (Figure 3, Table S3). No significant changes in indole glucosinolates were detected, while most tested T-DNA lines showed at least a tendency toward higher aliphatic glucosinolate levels, and five of these showed a significant increase in total aliphatic glucosinolate abundance. The respective lines contained insertions in genes coding for a vesicle associated membrane protein (VAMP721, At1G04750), a ubiquitinconjugating enzyme (UBC36, At1G16890), Calnexin 1 (CNX1, At5G61790) and two unknown membrane proteins (At2G32380, At1G44920). Strikingly, all five lines with significantly increased aliphatic glucosinolates contained mutations in genes found with the CYP83A1 bait (Figure 3). 


\section{A}
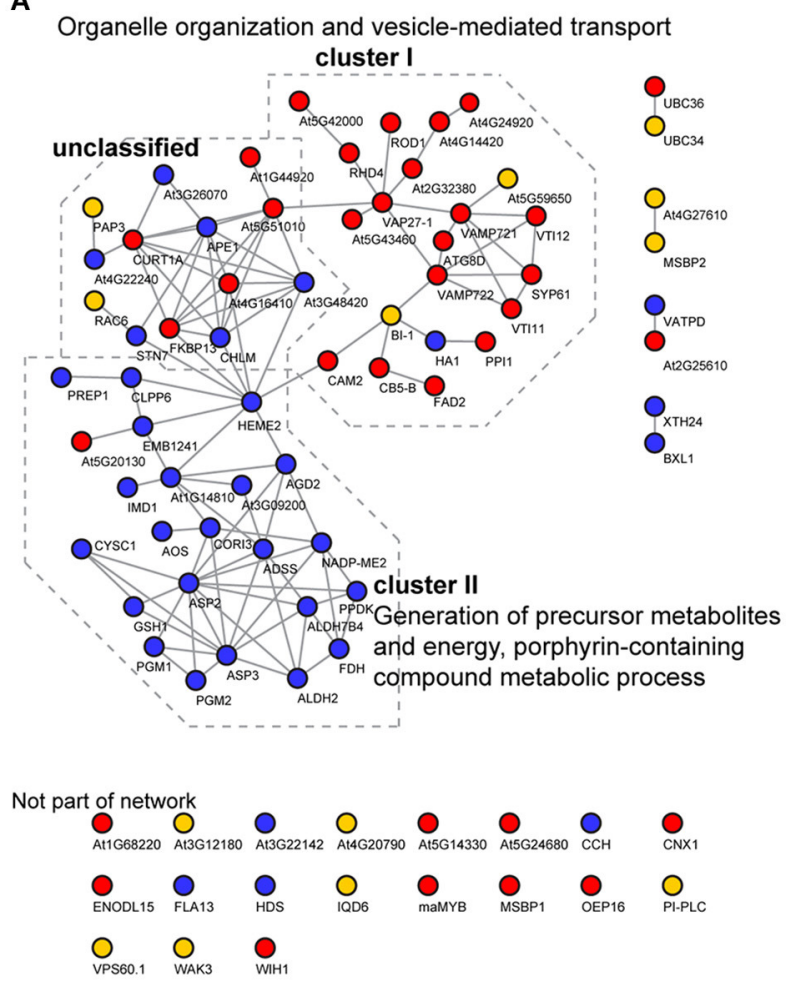

B

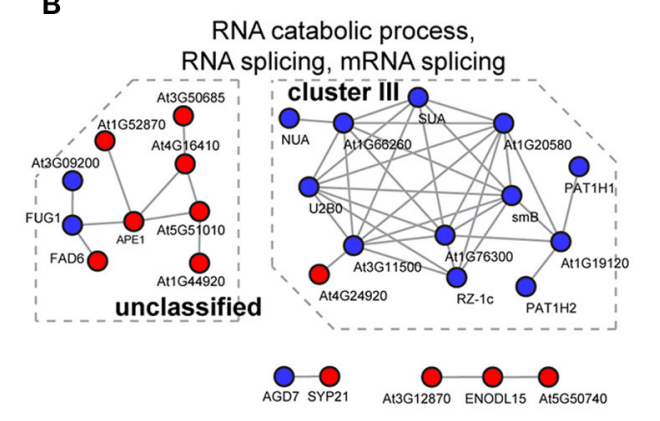

Not part of network

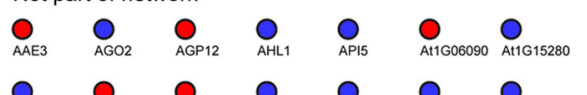

$\bigcirc_{A t 1 G 19880} \bigcirc_{\text {At1G28250 }} \bigcirc_{\text {At1G57765 }} \bigcirc_{\text {At1G67170 }} \bigcirc_{\text {At1G79090 }} \bigcirc_{\text {At2G04170 }} \bigcirc_{A t 2 G 20950}$

$\bigcirc_{A t 3 G 14750} \bigcirc_{A t 3 G 18280} \bigcirc_{A t 3 G 20550} \bigcirc_{A t 3 G 22142} \bigcirc_{A t 3 G 22430} \bigcirc_{A t 3 G 62200} \bigcirc_{A t 4 G 16830}$

$\bigcirc_{A t 5 G 14720} \bigcirc_{A t 5 G 19950} \bigcirc_{A t 5 G 42570} \bigcirc_{A t 5 G 43460} \bigcirc_{A t 5 G 47430} \bigcirc_{A t 5 G 51400} \bigcirc_{A t 5 G 52980}$

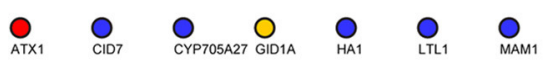

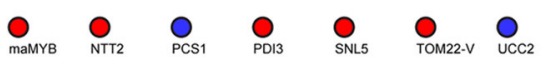

UPI

\begin{tabular}{|l} 
Source \\
$\bigcirc$ Yeast 2-hybrid $\bigcirc$ Co-IP $\bigcirc$ BioGrid
\end{tabular}

FIGURE 2 | Functional networks of CYP83A1 and CYP83B1 interactors. Network analysis was performed on interactors identified by yeast 2-hybrid, Co-IP and the BioGrid database using CYP83A1 (A) and CYP83B1 (B) as baits. The network was built using the STRING database with a threshold score of 0.4 (Szklarczyk et al., 2015). Emerging clusters were subjected to Gene Ontology enrichment, and the main enriched GO categories of the respective sub-clusters are displayed.

\section{Proteins Associated with Innate Immunity Interact with Glucosinolate Biosynthetic Enzymes}

Two homologous proteins annotated as HR-like lesion-inducing protein-related (At4G14420, At5G43460) were detected as interactors of CYP83A1 and, in the case of At5G43460, also of CYP83B1 in our yeast 2-hybrid screens. Strikingly, At5G43460 was found multiple times in all four of these screens, thus prompting us to investigate its interaction specificity and potential role in glucosinolate metabolism. In targeted assays, we re-tested the interaction between this protein and both CYP83 enzymes and investigated interactions with other glucosinolate biosynthetic enzymes, as well as the unrelated LargeT protein. We found that co-expressing At5G43460 with CYP83s, CYP79F2 or GGP1, but not CYP79F1 or LargeT enabled yeast cells to grow on selective medium (Figure 4). These results show that while the At5G43460 gene product interacts with several glucosinolate biosynthetic enzymes, it only activates the yeast 2 -hybrid system when co-expressed with certain baits. We were thus interested in whether this protein or its homologs affect glucosinolate accumulation.

The gene products of At5G43460 and At4G14420 are annotated as HR-like lesion-inducing proteins-related, as they contain an HR-like lesion-inducer domain (PFAM:PF05514/InterPro:IPR008637). However, no molecular function has yet been assigned to these proteins. The Arabidopsis genome encodes four additional homologous proteins that share the HR-like lesion-inducer domain, one of them, At1G04340, is a close homolog of At5G43460 and At4G14420. We named the gene products of At1G04340, At4G14420, and At5G43460 HR-like 1,4 , and 5 , respectively, according to their gene locations on the different chromosomes. At the amino acid level, HR-like 5 shares 83 and 52\% sequence identity with HR-like 1 and HR-like 4 , respectively. The three proteins form a subclade within the small family of HR-like proteins (Figure S6). The other three family members, At3G23175, At3G23180, and At3G23190 are located in close proximity on chromosome 3 and share between 19 and $24 \%$ amino acid identity with the members of the subclade with HR-like 1, 4, and 5. The high similarity of HR-like 1,4 , and 5 and their potential involvement in innate immunity prompted us to further investigate these proteins, despite the fact that mutations in individual genes did not appear to alter glucosinolate accumulation in the respective T-DNA insertion lines (Figure 3).

To address whether protein-protein interactions between CYP83 enzymes and HR-like proteins could fulfill a biological function in planta, we transiently expressed fluorophore-tagged versions of CYP83A1 and CYP83B1 as well as HR-like 1, 4, and 5 in Nicotiana benthamiana epidermis cells to determine their subcellular localization (Figure 5). The visible mesh structure and the signal surrounding, but not filling the nuclei, confirmed the predicted presence of CYP83 enzymes at the ER membrane 


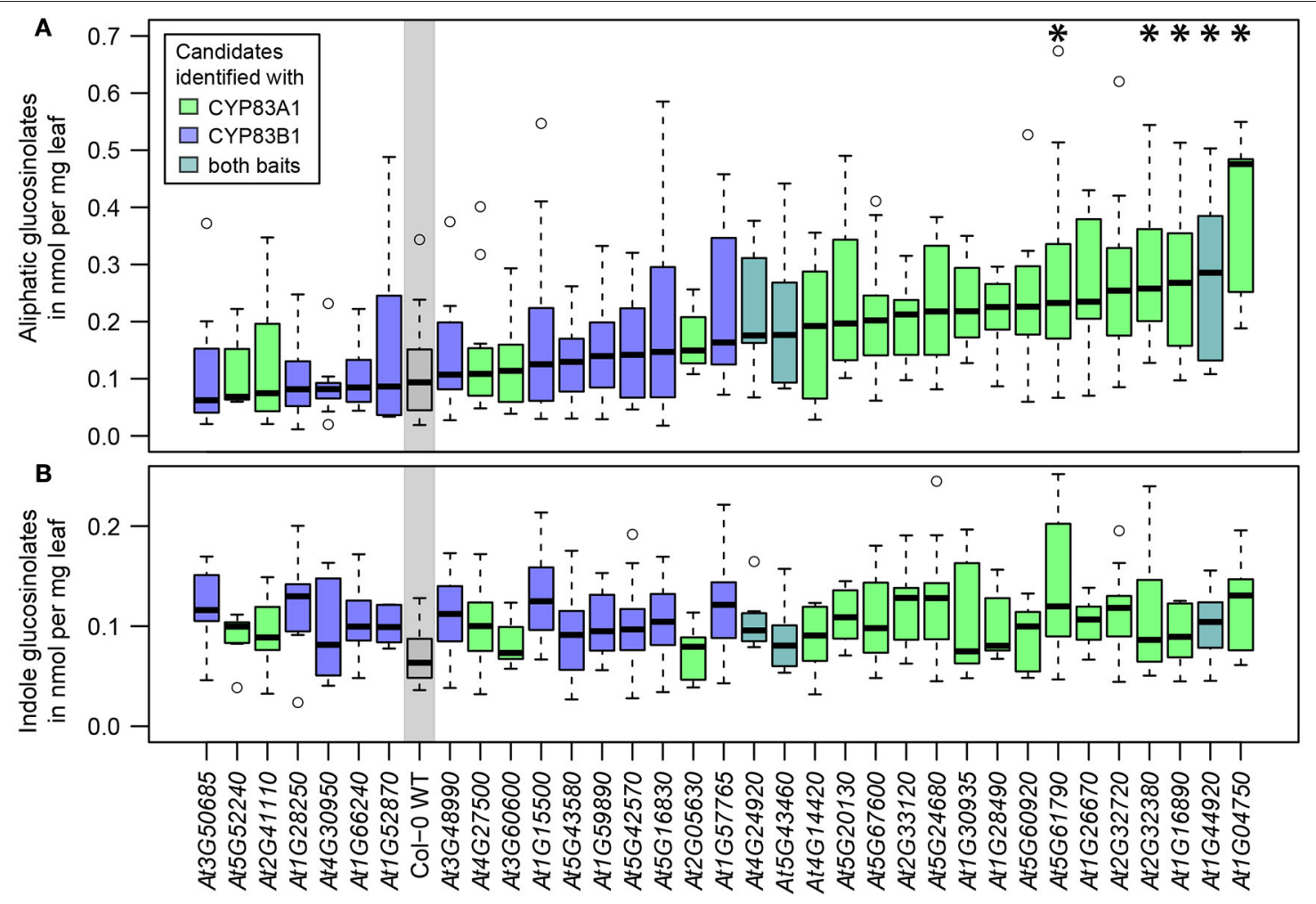

FIGURE 3 | Glucosinolate analysis of plants carrying mutant alleles of identified genes. (A) Total aliphatic glucosinolate levels. (B) Total indole glucosinolate levels. The genes of candidate interactors are sorted and plotted according to their median aliphatic glucosinolate content and the plot is color coded according to the baits with which the proteins were identified. Glucosinolate levels significantly deviating from wildtype (WT) levels are marked with * (Two-way ANOVA (Table S4) followed by post-hoc $t$-test, Holm-adjustment for multiple testing, $p<0.05, n=6-12$, Col-0 WT: $n=22$ ].

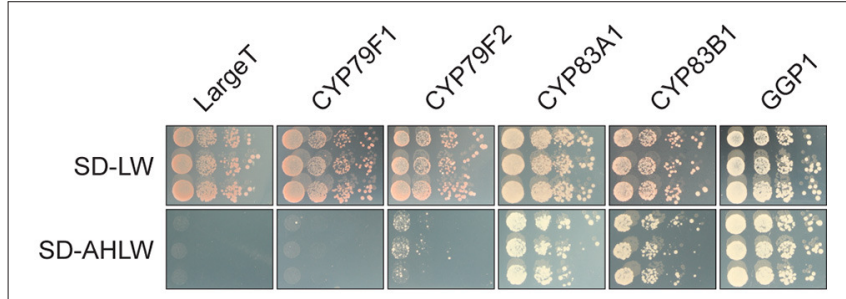

FIGURE 4 | The At5G43460 protein interacts with several glucosinolate biosynthetic enzymes. Combinations of At5G43460 and enzymes from the glucosinolate biosynthetic pathways or the unrelated control bait protein LargeT were analyzed in a yeast 2-hybrid assay. Growth control (SD-LW medium) and interaction assay (SD-AHLW medium) is depicted.

(Figures 5A,B). We observed the same pattern for all three investigated HR-like proteins (Figures $5 \mathbf{C}-\mathbf{E}$ ), indicating the presence of all five proteins in the ER membrane, a prerequisite for protein-protein interactions to occur in planta.

\section{HR-Like Proteins Have an Impact on Glucosinolate Accumulation}

We further investigated whether the identified HR-like proteins are functionally linked to glucosinolate accumulation by measuring glucosinolate concentrations in plants carrying mutations in one $(h r 1, h r 4, h r 5)$, two $(h r 14, h r 15, h r 45)$, or all three ( $h r 145)$ of the genes (Figure 6). As observed before for $h r 4$ and $h r 5$, the single mutant alleles did not result in a significant change in short-chained aliphatic, long-chained aliphatic, total aliphatic, indole or total glucosinolate content compared to wildtype. However, a specific and significant increase of $29-47 \%$ in short-chained aliphatic glucosinolates-i.e., glucosinolates derived from methionine with one, two or three additional methylene groups in the side chain-was observed in plants with two mutant alleles, and the triple insertion line hr145 showed an increase of $66 \%$ in these compounds. The absence of an observable effect in the single mutants and the apparent additive effect of multiple insertions in genes of different members of this family suggest a redundant action of HR-like 1, 4, and 5 on the accumulation of short-chained aliphatic glucosinolates.

\section{DISCUSSION}

In this study, we set out to identify protein interactors of the aliphatic and indole glucosinolate biosynthetic pathways using the pathway-specific enzymes CYP83A1 and CYP83B1 as baits in untargeted yeast 2-hybrid and Co-IP approaches. Our results highlight the potential interaction of the CYP83s with a large number of proteins. Noticeably, neither biosynthetic enzymes 


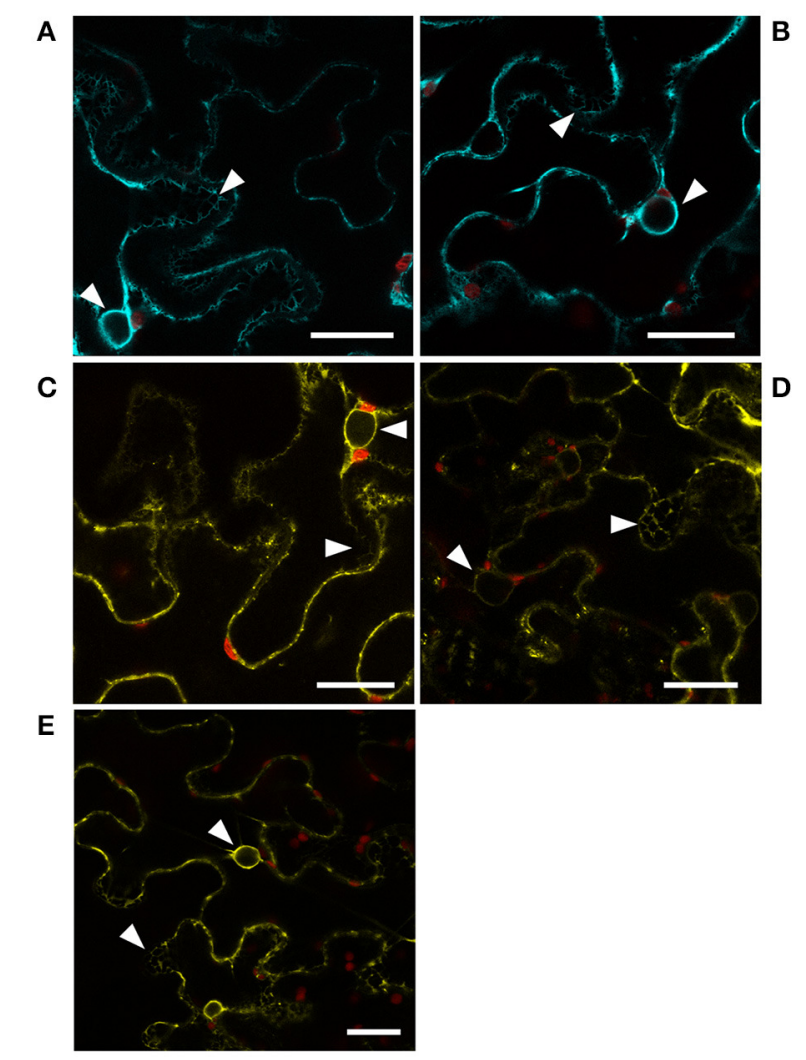

FIGURE 5 | Localization of CYP83 enzymes and HR-like proteins. Subcellular localization of (A) CYP83A1-mTurquoise2, (B) CYP83B1-mTurquoise2, (C) HR-like 1-mVenus, (D) HR-like 4-mVenus, and (E) HR-like 5-mVenus fusion proteins transiently expressed in Nicotiana benthamiana epidermis cells and observed by confocal laser scanning microscopy. mTurquoise 2 and mVenus fluorescence signal is represented by cyan and yellow, respectively, while red represents signal containing chlorophyll autofluorescence. White arrow heads point to signal from ER membranes in mesh-like structures and surrounding the nuclei. Scale bars: $25 \mu \mathrm{m}$.

from the pathways of the glucosinolate core structure nor any apparent scaffolding proteins or assembly chaperones were among the total of 75 (CYP83A1 bait) and 70 (CYP83B1 bait) putative interactors. Instead, our findings draw attention to the protein-protein interaction networks into which the biochemical pathways are embedded. Most interestingly, our results seem to indicate a link to innate immunity through interactions with proteins involved in cell death regulation and HR.

\section{Complementary Approaches Uncover Diverse, Comprehensive Networks of Interactors}

When interpreting results, it is crucial to consider technical strengths, but also biases and limitations of the chosen methods. In contrast to Co-IP, yeast 2-hybrid can detect interactions with very lowly abundant proteins, provided the respective sequence is present and successfully taken up by yeast cells. However, the employed cDNA libraries do not contain full coverage of the transcriptome. The choice of library can be critical (Brückner et al., 2009), as also suggested by the little overlap between our screens of different libraries with identical baits. Furthermore, the probability of a prey protein being successfully expressed in yeast increases with abundance of respective plasmids. Long transcripts are more likely to be incompletely reverse transcribed and thus lead to incomplete or misfolded protein fragments. In this study, the yeast 2-hybrid approach showed an additional bias toward membrane-bound proteins, which might in part be explained by the usage of membrane-anchored baits. Together, this leads to a technical bias toward shorter, membrane-bound proteins, encoded by transcripts of high abundance.

Frequent presence amongst colonies-as observed with HRlike 5 in the yeast 2-hybrid screens-can also be linked to interaction strength, as stronger interactions result in more successful colony formation events. Available transcript data (Winter et al., 2007) for this gene shows lower or comparable levels to the lowly expressed CYP83A1 and CYP83B1 in relevant tissues, suggesting that the gene is not enriched in our libraries. The relatively large number of candidate interactors that, in addition to interacting with both CYP83A1 and CYP83B1, also interacted with Large $\mathrm{T}$ suggests that some of the interactions are of promiscuous nature and appear between a broad range of proteins. Finally, in certain cases, interactions can involve the employed protein tags and thereby contribute to the relatively high false positive rate of yeast 2-hybrid screens reported in the literature (von Mering et al., 2002). Affinity enrichment methods such as Co-IP have the advantage that it is possible to detect indirect protein-protein interactions. Protein extraction itself, however, may disrupt unstable or otherwise transient protein complexes, particularly in the presence of detergents used to solubilize membrane proteins. This problem is further aggravated by the need of subsequent wash steps in order to reduce unspecific interactions of highly abundant proteinsoften photosynthetic proteins in plant samples (Smaczniak et al., 2012; Gupta et al., 2015). Losses due to adsorption during sample preparation favor hydrophilic proteins, and tryptic digest followed by MS-based proteomics favors longer proteins, which further contributes to methodical bias toward stable interactions between soluble, abundant proteins.

A relatively large proportion of the candidates detected in CoIP is predicted or has been determined to localize to plastids. It is important to note that predictions are not always correct and that even in cases where experimental evidence is available, the subcellular localization indicated in Tables 1-3 only reflects a consensus based on often conflicting findings. Fractions of protein populations can localize to different compartments, proteins could interact during protein translocation or be localized to the periphery of plastids where physical contact to ER membranes is possible. We thus decided not to disregard candidates based on their reported or predicted subcellular localization.

The question whether the observed interactions occur in planta, as well as their biological relevance and mode of action, can only be addressed on a case-by-case basis. When interpreting annotations and functional networks, it has to be considered that the underlying connectivity is often only based on predictions, 


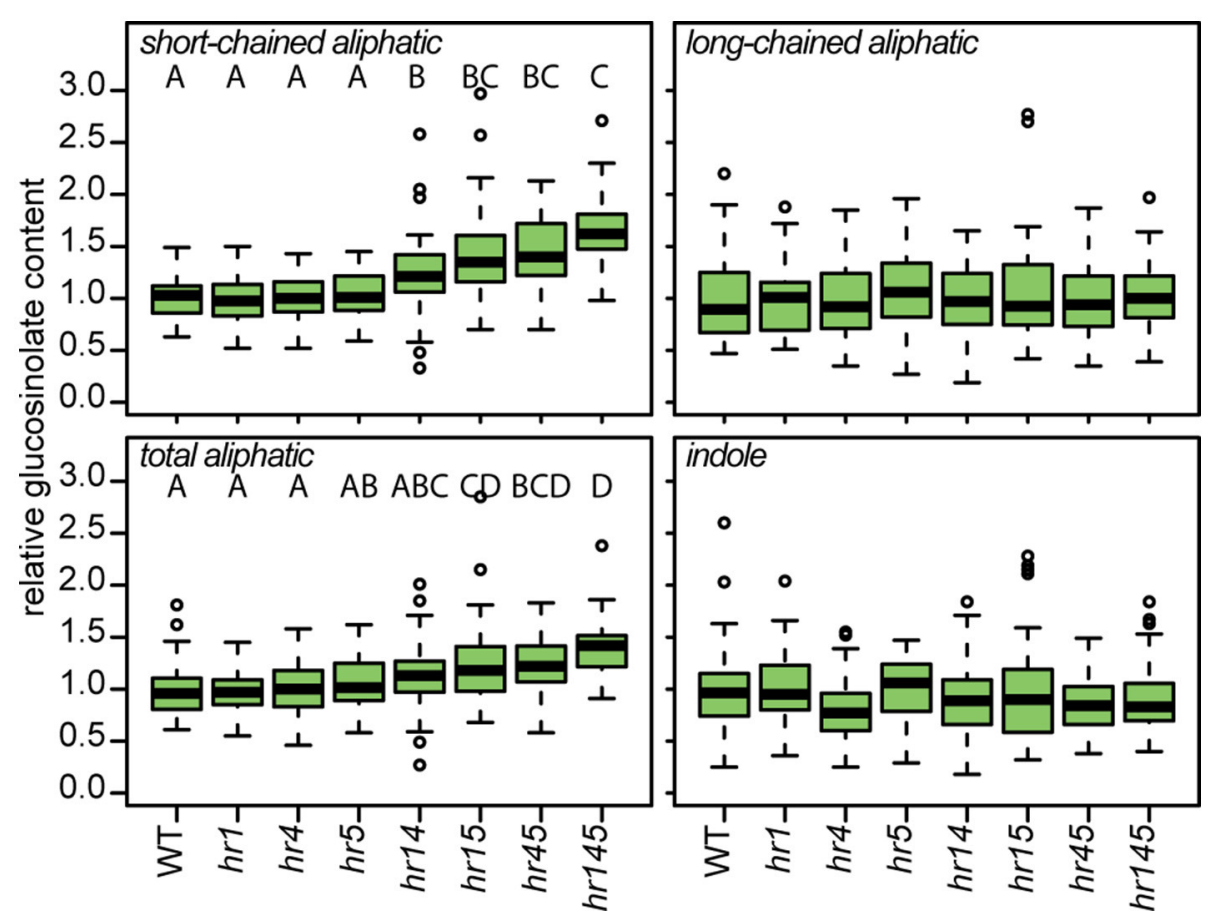

FIGURE 6 | HR-like genes influence the accumulation of a specific class of glucosinolates. Relative content of different classes of glucosinolates in 12-day-old plants carrying mutant alleles of one or several HR-like genes [Two-way ANOVA (Table S5) followed by post-hoc t-test, Holm-adjustment for multiple testing, $p<0.05$, $n=43-48]$.

and that large parts of the proteome are still unexplored. Many proteins lack proper annotation, as exemplified by the large group of unclassified proteins in our network analysis. It is estimated that as much as half of all eukaryotic proteins cannot be assigned a function or structure, and that especially shorter proteins belong to the so-called "dark proteome" with no assigned structural similarity to described proteins and generally little available knowledge about their functions (Perdigao et al., 2015).

In summary, the complementary strengths of the employed methods contribute to a high diversity of proteins found by our independent approaches, allowing for a more comprehensive view of the protein-protein interaction networks. At the same time, the sensitivity, the choice of experimental design as well as the inherent limitations and biases complicate comparisons to other experiments, which may explain the few previously reported interactors in our datasets.

\section{Network Analysis Allows Interpretation of Fragmentary Data}

Network analysis enables assessment of functional associations between proteins based on a variety of properties, including physical protein-protein interactions, co-expression, homology, and participation in pathways and biological processes (von Mering et al., 2005; Szklarczyk et al., 2015). In our network analysis, approximately two thirds of the candidates could be classified as belonging to one of three GO term enriched clusters.
The proteins in cluster I (organelle organization and vesiclemediated transport) were mainly identified by yeast 2-hybrid and contain putative interactors of CYP83A1. Cluster II consists of detected interactors of CYP83A1 as well, and almost exclusively of proteins identified in Co-IP experiments. The cluster contains proteins involved in the generation of precursor metabolites and energy and porphyrin-containing compound metabolic process. The interaction between a glucosinolate biosynthetic enzyme and these proteins is intriguing, as it could point to e.g. a feedback coupling of the pathway to the production of required precursors from primary metabolism. Cluster III is the only functionally enriched cluster of detected interactors of CYP83B1 and contains proteins involved in mRNA processing and splicing.

A possible explanation for the high number of membraneassociated proteins grouped into cluster I could be the spatial organization of glucosinolate pathways within the cell. The CYP83A1 enzyme localizes to the cytosolic face of the ER, while downstream enzymes of the pathways are soluble in the cytosol. Micro-compartments as formed by membrane invaginations or protein complexes at the membrane surface might fulfill a role in regulating local concentration of intermediates, and in effect promote substrate channeling and circumvent escape of instable or toxic intermediates, similar to what has been proposed for metabolons. Such micro-compartmentalization has been observed between the ER and plastids, where it allows for metabolic continuity between these compartments (Mehrshahi et al., 2013, 2014). Given that methionine chainelongation steps in aliphatic glucosinolate biosynthesis are 
catalyzed by plastidic enzymes, a similar mechanism could facilitate this pathway by allowing for an elegant way of feeding chain-elongated methionine from the plastids into the ER-associated cytosolic aliphatic glucosinolate core pathway. Micro-compartmentalization of glucosinolate pathways could also reflect subpopulations of the biosynthetic machinery for specific purposes. For instance, the ER-associated CYP83A1 and CYP83B1 were both found enriched in Co-IP experiments with the late endosomal vesicle marker ARA7 (Heard et al., 2015). This localization of the CYP83 enzymes away from the ER membrane could be promoted by interacting proteins as found in cluster I of our network, and possibly serve biosynthesis and storage of glucosinolates destined for vesicular export. Indeed, such exosomes containing components of the glucosinolatemyrosinase defense system were identified and proposed as an apoplastic storage space for antimicrobial compounds or their precursor molecules (Rutter and Innes, 2016). Intriguingly, these exosomes were enriched in stress-associated proteins, and their secretion was stimulated upon Pseudomonas syringae infection, suggesting a role in immunity (Rutter and Innes, 2016). Another example of micro-compartmental localization of glucosinolate biosynthesis is the indole glucosinolatemodifying enzyme CYP81F2 in epidermal cells, which in challenged cells shows a reticulate distribution, as well as focal accumulation at the site of microbial attack (Fuchs et al., 2016).

VAMP722 and VAMP721 that both have an established role in immunity (Yun et al., 2013; Kim et al., 2014), were also found as interactors of CYP83A1 and contribute to cluster I in the present study. Cluster I furthermore contains the known interactor BI1, a regulator of cell death. While we did not detect this interactor in any of our screens, several of our candidate interactors connected with this protein in the network analysis: the mentioned VAMP722 (At2G33120), HA1 (At2G18960), CAM2 (At2G41110), and CB5-B (At2G32720). BI1 interacts physically with CB5-B and calmodulins (IharaOhori et al., 2007; Kawai-Yamada et al., 2009), and these protein-protein interactions are proposed to enable BI1 to act on fatty acid modification and consequently fulfill its role in cell death suppression (Nagano et al., 2009). Both CB5-B and CAM2/AtCAL5-one of seven Arabidopsis calmodulins-were also found as interactors of CYP83A1 in our screens. The link of BI1 with CB5-B, CAM2, the fatty acid desaturase 2 (FAD2), as well as reduced oleate desaturation 1 (ROD1) is of particular interest, as lipid composition is a major cue in the onset of programmed cell death in response to stress (Liang et al., 2003; Nagano et al., 2009). The interplay between these proteins could explain the regulatory role of BI1, as lipid composition can be crucial for enzyme activity and complex formation in biochemical pathways (Laursen et al., 2016). An impact of lipid composition on glucosinolate metabolism, facilitated by CYP83A1's proximity to the respective protein complexes, seems plausible.

In conclusion, through network analysis, we were able to classify and group many of the detected interactors, and show that the interactors of CYP83A1 and CYP83B1-marker enzymes for aliphatic and indole glucosinolate biosynthesis-are functionally different, probably reflecting that aliphatic and indole glucosinolates function in different biological contexts.

\section{Linking Glucosinolate Metabolism with Hypersensitive Response/Innate Immunity}

The network containing BI1 suggests a link from glucosinolate metabolism to cell death regulation. BI1 has been directly linked to regulation of HR (Matsumura et al., 2003; KawaiYamada et al., 2009), which is triggered by pathogen effectors and induces a rapid programmed cell death reaction at the site of infection, thereby counteracting the spread of biotrophic pathogens (Wu et al., 2014). It is known that indole glucosinolates are essential for HR-mediated immunity (Bednarek et al., 2009; Clay et al., 2009; Johansson et al., 2014) and that intermediates and products of aliphatic glucosinolate biosynthesis play an essential role in interactions between plants and the pathogenic fungus Erysiphe cruciferarum (Weis et al., 2014). Additionally, a role for glucosinolates and derived metabolites, as well as other specialized metabolites, has been proposed in regulation of innate immunity responses (Bednarek, 2012). Based on our findings, as well as the interactions described in previous studies, we propose that an interaction network between proteins involved in innate immunity and glucosinolate biosynthesis links these defense strategies.

As novel candidates for such a link, we investigated members of a small family of proteins containing a domain associated with HR. These HR-like proteins were detected as interactors of both CYP83 enzymes in this study, and are further linked to HR via yeast 2-hybrid interactions with established HR regulators (Arabidopsis Interactome Mapping Consortium, 2011). For example, both HR-like 5 and HR-like 1 interact with ANAC089 (associated with HR and ER stress-induced cell death, Yang et al., 2014) and VAP27 (an interactor of ACD11, a known regulator of HR, Petersen et al., 2009). HR-like proteins interact with a multitude of proteins and may-in concert with the many other glucosinolate pathway interactors-lead to an alteration in glucosinolate metabolism in response to a defense cue. This is demonstrated by our analysis of mutant alleles of three HRlike proteins. Plants with insertions in multiple HR-like genes showed an apparent additive increase in the accumulation of short-chained aliphatic glucosinolates.

Phenotypic analyses of genetic perturbations of candidate genes are a versatile approach to link genes to potential biological functions. In our sample of T-DNA insertion lines in genes of 34 proteins found to interact with either CYP83A1 or CYP83B1, a considerable proportion showed a trend, some a significant increase in aliphatic glucosinolate levels under our experimental conditions (Figure 3). It has been reported earlier that glucosinolate metabolism is generally sensitive to mutations of leaf-expressed genes (Chan et al., 2011). It can therefore be assumed that the observed impact on glucosinolate metabolism can partially be explained by this background influence, caused by any genetic perturbation that affects the plant's physiology. However, the correlation of interactors detected with CYP83A1 as bait and the changes in aliphatic glucosinolate accumulation are an indication that the detected protein-protein interactions 
indeed have an impact on glucosinolate accumulation. It is known that many glucosinolate phenotypes are dependent on environmental factors (Gigolashvili et al., 2008; Frerigmann and Gigolashvili, 2014; Li et al., 2014; Burow, 2016), and especially interactors related to defense signaling might only reveal condition-specific phenotypes. The overall trend in our highthroughput phenotypic analysis, along with the observations in plants carrying mutations in HR-like genes, point toward a role of protein-protein interactions as negative regulators of basal aliphatic glucosinolate levels. Perturbations of the interaction network upon attack (possibly mimicked by the genetic perturbations in T-DNA mutant lines) could give a rapid boost in glucosinolate production, even before the biosynthetic machinery has been upregulated.

Using complementary untargeted methods, we discovered a large number of novel candidate interactors of glucosinolate biosynthetic enzymes. Through network analysis, we identified networks of interacting proteins involved in biological processes that were clearly distinct between the marker of the aliphatic glucosinolate pathway, CYP83A1, and the marker of the indole glucosinolate pathway, CYP83B1. Our results suggest that-rather than forming a stable metabolon structure-the glucosinolate pathways may assemble stochastically through a multitude of transient interactions in a highly organized microenvironment. Based on the interaction networks, we propose a protein level link between innate immunity and glucosinolate metabolism. Future investigations will unravel the molecular link between HR-like proteins and aliphatic glucosinolates, as a functional link to HR seems plausible.

\section{EXPERIMENTAL PROCEDURES}

\section{Bait Cloning and Evaluation}

The coding sequences of CYP83A1 (At4G13770) and CYP83B1 (At4G31500) were PCR amplified using the Phusion U DNA polymerase (Thermo Scientific) and A. thaliana, ecotype Columbia-0 cDNA as template with primer pairs 83A1_Sfi_F/83A1_Sfi_nostop_R and 83A1_Sfi_F/83B1_Sfi_nostop_R, respectively. The PCR products as well as the pDHB1 bait vector (Dualsystems Biotech) were digested with SfiI (New England Biolabs) and the CYP83A1 and CYP83B1 coding sequences ligated to the vector backbone (Figure S7). The proper fusion and insertion of all sequences was confirmed by DNA sequencing. Oligonucleotide sequences are listed in Table S1.

Saccharomyces cerevisiae strain NMY51 (Dualsystems Biotech, MATa his $\Delta 200$ trp1-901 leu2-3,112 ade2 LYS2::(lexAop) $)_{4}$-HIS3 ura3::(lexAop) - $_{8}$ lacZ ade2::(lexAop) 8 $^{-}$ ADE2 GAL4) was transformed with the pDHB1-CYP83A1 and pDHB1-CYP83B1 bait plasmids by the LiAc/PEG method (Gietz and Schiestl, 2007) and selected for the presence of the plasmid on synthetic complete dropout medium (Sunrise Science Products) with yeast nitrogen base (Duchefa) and glucose, lacking leucine (SD-L), generating the two bait carrying strains for the library screens. These strains were tested for bait functionality and background growth by transformation with the control preys pPR3-N (empty prey vector), NubI (positive control prey) and the unrelated prey $\triangle \mathrm{P} 53$, interactor of the control bait LargeT (SV40 large T antigen) (Möckli et al., 2007). The selection stringency was titered by the addition of increasing concentrations 3-Amino-1,2,4-Triazole (3AT, Sigma) to SD media. 3AT is a competitive inhibitor of the HIS3 reporter that increases the stringency of the histidine selection system and can be used to enable screens with moderately autoactivating bait proteins (Snider et al., 2010).

\section{Library Construction}

Two independent cDNA libraries based on the pPR3-N prey vector (Dualsystems Biotech) were generated from centers of rosettes of 3-week-old plants grown on soil and hypocotyls from 10-day-old seedlings grown on Murashige and Skoog medium including vitamins (M0222, Duchefa), respectively. The plant tissues were collected on ice and subsequently frozen in liquid nitrogen. Total RNA was extracted from $30 \mathrm{mg}$ of hypocotyls and $60 \mathrm{mg}$ of nodes using the Qiagen RNeasy plant mini kit. The obtained RNA was analyzed using an Agilent Bioanalyzer 2100. Double stranded cDNA was produced with Sfi-oligo-dT and Sfirandom primers using the Clontech SMART ${ }^{\circledR}$ cDNA library construction kit following the manufacturer's instructions. The cDNA and the empty pPR3-N vector were digested with SfiI, the linearized plasmid DNA was treated with calf intestine phosphatase (Sigma Aldrich) and purified by gel electrophoresis and the QIAquick Gel Extraction Kit (Qiagen). 100 ng of the SfiI digested and dephosphorylated pPR3-N vector was ligated with T4 DNA ligase (New England Biolabs) to $0.5,1$, or $1.5 \mu \mathrm{L}$ of SfiIdigested cDNA. After incubation overnight, the ligation mixture was sodium acetate/ethanol precipitated and resuspended in $5 \mu \mathrm{L} \mathrm{H} \mathrm{H}_{2} \mathrm{O} .25 \mu \mathrm{L}$ of MegaX DH10B ${ }^{\mathrm{TM}} \mathrm{T} 1 \mathrm{R}$ Electrocomp ${ }^{\mathrm{TM}}$ Cells (Invitrogen) were used for transformation with $1 \mu \mathrm{L}$ of ligation mix. After $1 \mathrm{~h}$ of recovery in recovery medium, dilutions of the transformation mixtures were plated on LB agar plates containing $100 \mu \mathrm{g} / \mathrm{mL}$ Ampicillin to determine the complexity of the libraries. The most complex libraries contained $3.8 \times 10^{5}$ and $4.4 \times 10^{5}$ transformants for the node- and hypocotyl library, respectively, and were selected for the untargeted yeast 2-hybrid screens. The node primary library was diluted to $40.6 \mathrm{~mL}$ and the hypocotyl primary library was diluted to $51.2 \mathrm{~mL}$ with $\mathrm{LB}$ medium. The cell suspensions were plated on $12 \mathrm{~cm}$ square LB agar plates containing $100 \mu \mathrm{g} / \mathrm{mL}$ Ampicillin in aliquots of 150 $\mu \mathrm{L}$ and the plates were incubated for $18-20 \mathrm{~h}$ at $37^{\circ} \mathrm{C}$. Plasmids from 12 randomly chosen colonies for each library were extracted and digested with SfiI. Insert sizes of between ca. $600 \mathrm{bp}$ and ca. 1,650 bp were observed for both libraries. All colonies were then resuspended in $3 \mathrm{~mL}$ of LB medium containing $25 \%$ glycerol per plate, scraped off the agar with a Drigalski spatula and combined to yield the node and hypocotyl libraries, respectively. Plasmids were isolated from $1.5 \mathrm{~mL}$ of each library using an alkaline lysis plasmid extraction protocol.

\section{Untargeted Yeast 2-Hybrid Screens}

Yeast cells containing the bait proteins were transformed with the prey libraries using the LiAc/PEG method (Gietz and Schiestl, 2007) and following the instructions of the Dualsystems Biotech DUALhunter kit manual. Dilutions of the library transformation 
mixtures were plated on SD-LW medium to determine the transformation efficiency (Table S2) and the remaining mixtures were plated on synthetic complete dropout medium lacking adenine, histidine, leucine and tryptophan (SD-AHLW) and containing $1 \mathrm{mM}$ of $3 \mathrm{AT}$ in the case of CYP83A1 and incubated for up to 6 days at $30^{\circ} \mathrm{C}$. Cells from growing colonies were allowed to grow in liquid SD-LW medium and the containing plasmids extracted and used for E.coli transformation. Prey plasmids were amplified in E. coli, extracted and sequenced to identify insert sequences. The sequences were analyzed by the basic local alignment search tool (BLAST) of The Arabidopsis Information Resource (TAIR, www.arabidopsis.org).

\section{Cloning of Prey Constructs for Pairwise Interaction Analysis}

For high throughput cloning of sequences into the pPR3-N prey vector, a USER ${ }^{\mathrm{TM}}$ (Nour-Eldin et al., 2010) compatible version of the vector was created by SfiI digestion and insertion of a USER $^{\mathrm{TM}}$ cassette (oligo sequences USER_F and USER_R). The coding sequences of prey genes were amplified by specific primers including USER ${ }^{\mathrm{TM}}$ sites (Table S1) and inserted into the vector by $\mathrm{USER}^{\mathrm{TM}}$ cloning (Figure S7). All sequences were confirmed by DNA sequencing.

\section{Drop Test}

To confirm pairwise interactions, yeast cells were co-transformed with bait and prey plasmids by the LiAc/PEG method (Gietz and Schiestl, 2007) and selected for the presence of both plasmids on SD-LW medium. Cells from three individual colonies were grown overnight in liquid SD-LW medium, sedimented by centrifugation, resuspended in sterile water and brought to an $\mathrm{OD}_{600}$ of 0.5 . Dilution series of $10^{-1}-10^{-4}$ were prepared in sterile water and $5 \mu \mathrm{L}$ of each dilution was dropped on plates containing SD-LW medium or SD-AHLW, containing $1 \mathrm{mM}$ $3 \mathrm{AT}$ in the case of CYP83A1 baits. The plates were incubated for 3 days at $30^{\circ} \mathrm{C}$ before analyzing yeast growth.

\section{Glucosinolate Analysis}

The SALK T-DNA insertion lines analyzed in this study are listed in Table S3. Glucosinolates were extracted from leaves of 3-week-old plants cultivated in a randomized setup on soil in a growth chamber with constant long day light conditions (16 h light, $100-140 \mu \mathrm{E} /\left(\mathrm{m}^{2} \mathrm{~s}\right), 21^{\circ} \mathrm{C}, 70 \%$ relative humidity) as desulfo-glucosinolates. For the analysis of single, double and triple insertion lines in HR-like genes, plants were grown on vertical plates containing Murashige and Skoog medium including vitamins (M0222, Duchefa) under the same conditions for 12 days before desulfo-glucosinolate extraction. Desulfo-glucosinolates were then analyzed by UHPLC/TQ-MS on an Advance ${ }^{\mathrm{TM}}$-UHPLC/EVOQ ${ }^{\mathrm{TM}}$ Elite-TQMS instrument (Bruker) equipped with a $\mathrm{C} 18$ reversed phase column (Kinetex $1.7 \mu \mathrm{XB}-\mathrm{C} 18,10 \mathrm{~cm} \times 2.1 \mathrm{~mm}, 1.7 \mu \mathrm{m}$ particle size, Phenomenex) as described previously (Jensen et al., 2015; Crocoll et al., 2016).

\section{Statistical Analysis}

We used R version 3.2.0 (2015-04-16) for statistical analysis (R Core Team, 2015). We tested the A. thaliana Columbia-0 wildtype and the different transgenic lines with the ANOVA function for the following linear model for effects on total indole or aliphatic glucosinolates separately: Indole glucosinolates or aliphatic glucosinolates = genotype + tray (Table S4). Specific differences between genotypes and the wildtype were tested post-hoc using the pairwise.t.test function with a Holmadjustment for multiple testing. For the analysis of insertions in HR-like genes, glucosinolate contents were normalized to corresponding wildtype levels and tested with the following linear model: Aliphatic, short- or long-chained aliphatic or indole glucosinolates $=$ genotype + experiment (Table S5). Specific differences between genotypes were tested post-hoc using the pairwise.t.test function with a Holm-adjustment for multiple testing.

\section{Functional Enrichment of Gene Ontology Categories}

Enrichment of functional categories was performed using PANTHER (version 11.1) (Mi et al., 2017) with the A. thaliana PANTHER GO-Slim biological process annotation released 2016-10-24. Unless specified otherwise, the whole genome list was used as reference list and, where possible, multiple testing correction was done with Bonferroni. GO terms with $p<0.05$ were considered as over-represented.

\section{Sequence Alignment and Phylogenetic Tree Construction}

Coding sequences of the six HR-like proteins At5G43460, At4G14420, At1G04340, At3G23175, At3G23180, and At3G23190 were retrieved from The Arabidopsis Information Resource (TAIR, www.arabidopsis.org) and aligned using the create alignment function of CLC Main Workbench (Version 7.6.1, Quiagen Aarhus A/S). The identity at the amino acid level was obtained by aligning the corresponding protein sequences and the pairwise comparison function of the program. The coding sequences were aligned using the ClustalW algorithm in JalView2 (Waterhouse et al., 2009) and a phylogenetic tree was generated using percentage identity from the Gblocks processed alignment.

\section{Construction and Stable Expression of Fluorophore-Tagged Proteins in Arabidopsis}

To introduce the coding sequence of mVenus (Nagai et al., 2002) into the pCambia1300U plant expression vector (Nour-Eldin et al., 2006), the sequence was amplified with primer pair USER_XFP_F/USER_XFP_R, the forward primer containing the sequence of a USER ${ }^{\mathrm{TM}}$ cassette in front of the $5^{\prime}$ end of the coding sequence of the fluorescent protein. The PCR product was then introduced into the PacI and Nt.BbvCI digested pCambia1300U vector by $\mathrm{USER}^{\mathrm{TM}}$ cloning. The promoter sequences including upstream genomic sequences and the $5^{\prime}$ UTR of CYP83A1 and CYP83B1 (1,033 and 1,001 
bp upstream of the start codon, respectively) were amplified from genomic DNA of $A$. thaliana ecotype Columbia-0 with the primer pairs Pro_83A1_F/Fus_Pro_83A1_R and Pro_83B1_F/Fus_Pro_83BA1_R. The corresponding coding sequences were amplified from $A$. thaliana ecotype Columbia- 0 cDNA with primer pairs Fus_83A1_F/83A1_Nostop_R and Fus_83B1_F/83B1_Nostop_R. The promoter and coding sequences were fused by USER ${ }^{\mathrm{TM}}$ fusion (Geu-Flores et al., 2007) and inserted into the USER ${ }^{\mathrm{TM}}$ cassette of the opened pCambia1300-mVenus vector. The proper fusion and insertion of sequences was confirmed by DNA sequencing. Oligonucleotide sequences are listed in Table S1. The plasmids were introduced into Agrobacterium tumefaciens strain GV3101 and $A$. thaliana ecotype Columbia- 0 was transformed by the floral dip method (Clough and Bent, 1998). Transgenic plants were selected by germination on $1 \%$ agar plates ( $1 / 2$ Murashige and Skoog medium including vitamins, M0222, Duchefa, containing $100 \mu \mathrm{g} / \mathrm{mL}$ Hygromycin, H0192.0001, Duchefa) and cultivated in a growth chamber with constant long day light conditions (16 h light, $100-140 \mu \mathrm{E} /\left(\mathrm{m}^{2} \mathrm{~s}\right), 21^{\circ} \mathrm{C}, 70 \%$ relative humidity). For each construct, 5 individual transgenic lines were analyzed for their global expression pattern by fluorescence microscopy. No differences in expression patterns were observed and one representative line was chosen for the following work. The same transgenic plant lines carrying PrCYP83A1:CYP83A1$\mathrm{mVenus}$ or PrCYP83B1:CYP83B1-mVenus were also used in $\mathrm{Xu}$ et al. (2016).

\section{Construction and Transient Expression of Fluorophore-Tagged Proteins in Tobacco}

For the expression of the CYP83A1, CYP83B1, HR-like 1, 4, and 5 coding sequences in Nicotiana benthamiana, constructs were generated as follows. A plant expression cassette containing a ubiquitin (UBQ10) promoter and a RBC terminator were PCR amplified and inserted into the pEAQ-HT (Sainsbury et al., 2009) vector backbone. The coding sequences of mTurquoise 2 (Goedhart et al., 2012) or mVenus were inserted into the resulting vector (Andersen et al., 2016). The respective coding sequences were PCR amplified with primer pairs CYP83A 1_FW/CYP83A1_NSR, CYP83B1_FW/CYP83B1_NSR, At1G0 4340_FW/At1G04340_NSR, At4G14420_FW/At4G14420_NSR, and At5G43460_FW/At5G43460_NSR (Table S1) and inserted into the USER ${ }^{\mathrm{TM}}$ cassette of the fluorescent protein tagging vectors. All sequences were verified by DNA sequencing. Agrobacterium tumefaciens strain GV3101 was transformed with plasmids carrying the sequences of fluorophore-tagged fusion proteins and grown overnight at $28^{\circ} \mathrm{C}$ and $220 \mathrm{rpm}$ in YEP medium containing the appropriate antibiotics. Cells were sedimented by centrifugation and resuspended in infiltration buffer (10 mM MES, $10 \mathrm{mM} \mathrm{MgCl}_{2}$ and $100 \mu \mathrm{M}$ acetosyringone (3,5-Dimethoxy-4-Hydroxyacetophenone, Sigma-Aldrich), $\mathrm{pH}$ 5.6). After $2 \mathrm{~h}$ incubation at room temperature and $150 \mathrm{rpm}$ agition, the cell suspensions were brought to an $\mathrm{OD}_{600}$ of 0.05 with infiltration buffer and infiltrated into the abaxial air space of leaves of Nicotiana benthamiana plants grown in small pots of $5.5 \mathrm{~cm}$ diameter in a green house at $24^{\circ} \mathrm{C}$ (day) and $18^{\circ} \mathrm{C}$ (night) with 50-60\% humidity for approximately 3-4 weeks (to 4-6 leaves stage).

\section{Microscopy}

Forty-eight hour after infiltration, confocal laser scanning microscopy was carried out on Nicotiana benthamiana leaves on a Leica SP5-X confocal microscope equipped with a HCX PL APO CS 63.0x1.20 WATER (1.2 numerical aperture, 63X magnification) objective. mVenus was excited at $514 \mathrm{~nm}$ and emission was collected at $524-550 \mathrm{~nm}$. mTurquoise 2 was excited at $458 \mathrm{~nm}$ and emission was detected at $468-518 \mathrm{~nm}$. Chlorophyll autofluorescence was collected at 650-750 nm.

\section{Plant Growth Conditions for Pulldowns}

Plants of the transgenic Arabidopsis lines expressing PrCYP83A1:CYP83A1-mVenus or PrCYP83B1:CYP83B1$\mathrm{mVenus}$ were grown on horizontal $1 \%$ agar plates ( $1 / 2$ Murashige and Skoog medium including vitamins, M0222, Duchefa, containing $100 \mu \mathrm{g} / \mathrm{mL}$ Hygromycin, H0192.0001, Duchefa), alongside wildtype (Columbia-0) plants (no selection). Seeds were cold-stratified for 3 days before growing for 14 days in climate chambers with constant long day light conditions (16 h light, $100-140 \mu \mathrm{E} /\left(\mathrm{m}^{2} \mathrm{~s}\right), 21^{\circ} \mathrm{C}, 70 \%$ relative humidity).

\section{Co-immunoprecipitation}

For each of the three independent experiments, three replicates of the three genotypes (wildtype, PrCYP83A1:CYP83A1mVenus and PrCYP83B1:CYP83B1-mVenus) were prepared by harvesting $2-4 \mathrm{~g}$ seedlings per replicate. These seedlings were flash frozen in liquid nitrogen and ground using mortar and pestle. The resulting powder was mixed $(1: 1 \mathrm{w} / \mathrm{v})$ with extraction buffer [ $150 \mathrm{mM}$ Tris $\mathrm{pH} 7.5,1 \mathrm{mM}$ EDTA, $150 \mathrm{mM}$ $\mathrm{NaCl}, 10 \%$ glycerol, $10 \mathrm{mM}$ DTT, 1\% IGEPAL CA630 (18896, Sigma-Aldrich), 2x Protease Inhibitor (11 873 580, Roche)]. After thorough mixing, the samples were incubated $30 \mathrm{~min}$, shaking at $4^{\circ} \mathrm{C}$. Samples were then spun at $13,000 \mathrm{~g}$ for $20 \mathrm{~min}$ at $4^{\circ} \mathrm{C}$. The resulting supernatants were mixed with $20 \mu \mathrm{L}$ of GFPtrap magnetic beads (Chromotek), which had been pre-equilibrated in extraction buffer. The samples were then incubated overnight at $4^{\circ} \mathrm{C}$, with "end-over-end" rotation. Magnetic separation of beads and supernatant were carried out on ice and the supernatants were discarded, before performing 3 washes with $400 \mu \mathrm{L}$ wash buffer each $(150 \mathrm{mM} \mathrm{NaCl}, 50 \mathrm{mM}$ TrisHCl, $\mathrm{pH}$ 8.0, 0.25\% IGEPAL CA630). Samples were eluted using $30 \mu \mathrm{L} 4 \%$ (w/v) SDS, $100 \mathrm{mM}$ Tris/HCl pH 8.2, 0.1 M DTT) and heating to $95^{\circ} \mathrm{C}$ for $5 \mathrm{~min}$ before magnetic separation of beads and elution.

\section{Protein Digest}

Each individual elution was mixed with $200 \mu \mathrm{L}$ UA buffer $(8 \mathrm{M}$ urea in $100 \mathrm{mM}$ TrisHCl, $\mathrm{pH}$ 8.2) before being loaded onto a centrifugal filter unit (Microcon-30 kDa Centrifugal Filter Unit Ultracel-30 membrane, Millipore MRCF0R030) and then spun at $14,000 \mathrm{~g}$ for $20 \mathrm{~min}$ at room temperature. The flow-through was discarded and $200 \mu \mathrm{L}$ of UA buffer were added to wash the filter by centrifugation at the above mentioned settings. Then, $100 \mu \mathrm{L}$ of $0.05 \mathrm{M}$ iodoacetamide (in UA buffer) were applied and incubated for $5 \mathrm{~min}$ before centrifugation. The filters were 
washed three times with $100 \mu \mathrm{L}$ UA buffer and twice with $100 \mu \mathrm{L}$ of $0.5 \mathrm{M} \mathrm{NaCl}$. Each filter was then moved to a new collection tube and $120 \mu \mathrm{L} 50 \mathrm{mM}$ ammonium bicarbonate with $3 \mu \mathrm{g}$ trypsin (Promega, V5073) were added to the filters, which were then shaken at $600 \mathrm{rpm}$ for $1 \mathrm{~min}$. The filters were incubated overnight at room temperature and the flow-through was eluted by centrifugation at $14,000 \mathrm{~g}$ for $20 \mathrm{~min}$. Flow-throughs were acidified ( $\mathrm{pH}<2.5)$ using $10 \%$ trifluoroacetic acid. Samples were then diluted in buffer S-A (2\% acetonitrile, $0.1 \%$ formic acid) up to $1.5 \mathrm{~mL}$ and spun at $20,000 \mathrm{~g}$ for $15 \mathrm{~min}$. The supernatants were each applied to a Sep-Pak column (Waters, WAT023590), pre-equilibrated using first $1 \mathrm{~mL}$ of buffer S-B (65\% acetonitrile, $0.1 \%$ formic acid) and then $1 \mathrm{~mL}$ of buffer S-A. Three washes with $1 \mathrm{~mL}$ of buffer S-A were carried out before eluting using two applications of $0.5 \mathrm{~mL}$ buffer $\mathrm{S}-\mathrm{B}$. The eluted samples were completely dried in a vacuum centrifuge, after which they were stored at $-20^{\circ} \mathrm{C}$ until further analysis.

\section{Mass Spectrometric Measurements}

Mass spectrometry measurements were performed on a LTQ OrbiTrap Velos mass spectrometer (Thermo Fisher) coupled to a NanoLC-ultra (Eksigent) using electrospray ionization. A 15$\mathrm{cm}$ capillary column, which was heated to $50^{\circ} \mathrm{C}$ and packed with $15 \mathrm{~cm} \mathrm{C18}$ beads with a diameter of $3 \mu \mathrm{m}$ and a pore size of $100 \AA$ was used for LC separation. Peptides were loaded on the column with a flow rate of $300 \mathrm{~nL} / \mathrm{min}$ for $20 \mathrm{~min}$ and eluted with a flow rate of $300 \mathrm{~nL} / \mathrm{min}$ for $65 \mathrm{~min}$ by an increasing gradient from $3 \%$ acetonitrile to $50 \%$ acetonitrile. The FT OrbiTrap was used for obtaining full scans at a range of 300-1,700 mass/charge, followed by MS/MS scans of the 20 highest parent ions. Dynamic exclusion was enabled at a duration of $45 \mathrm{~s}$.

\section{Interpretation of MS/MS Spectra}

The obtained raw spectra were transformed to mgf data format and searched against the TAIR10 database (download on January 17th, 2011; Lamesch et al., 2012) with concatenated decoy database and supplemented with common contaminants (71,032 entries) using the Mascot algorithm (version 2.3.02) (Mascot Science). The search parameters used were: mass = monoisotopic, requirement for tryptic ends, 2 missed cleavages allowed, precursor ion tolerance $= \pm 10 \mathrm{ppm}$, fragment ion tolerance $= \pm 0.5 \mathrm{Da}$, variable oxidation of methionine (M, PSIMOD name: oxidation, mono $\Delta=15.995)$, protein $\mathrm{N}$-terminal acetylation (protein N-term, PSI-MS name: acetyl, mono $\Delta=$ $42.010565 \mathrm{Da})$ and conversion of N-terminal glutamine into pyroglutamic acid (N-term Q, PSI-MS name: Gln $\rightarrow$ pyro-Glu, mono $\Delta=-17.026549 \mathrm{Da})$, and static carbamidomethylation of cysteine (C, PSI-MS name: carbamidomethyl, mono $\Delta=$ 57.0215) (Svozil and Baerenfaller, 2017). Peptide spectrum assignments with a Mascot score higher than the ion score that indicates identity or extensive homology $(p<0.05)$ in the respective sample were loaded into the pep2pro database. Peptides matching to known contaminants or to several proteins were excluded from further analyses, unless they belong to a different splice variant of the same protein or to a different locus, which shares exactly the same amino acid sequence (Baerenfaller et al., 2011; Hirsch-Hoffmann et al., 2012). For calculating the false discovery rate (FDR) the number of reverse hits was divided by the number of forward hits times $100 \%$. All mass spectrometry proteomics data have been deposited to the ProteomeXchange Consortium (www.proteomexchange.org) via the PRIDE partner repository (Vizcaino et al., 2013) with the dataset identifiers PXD005585 and 10.6019/PXD005585. Furthermore, the data can be found in the pep2pro database at www.pep2pro.ethz.ch. Proteins were quantified by spectral counting and only proteins identified with at least five spectra were taken into consideration. Enriched proteins had to have at least 5 times more spectrum counts in the respective GFPtrap line compared to the wildtype control.

\section{Bioinformatics Resources and Analysis of Interaction Candidates}

Descriptions and predicted transmembrane domains of the identified proteins (from both yeast 2-hybrid and Co-IP) were retrieved from The Arabidopsis Information Resource (TAIR, www.arabidopsis.org), via the bulk retrieval function. Molecular weights, amino acid lengths, isoelectric points, GRAVY scores and subcellular localization were retrieved from SUBA3 (Hooper et al., 2014) and the UniProt databases (Bateman et al., 2015; Boutet et al., 2016). Published interactions of CYP83A1 (At4G13770) and CYP83B1 (At4G31500) were extracted from the BioGrid database (Chatr-Aryamontri et al., 2015) (Table S6).

Our interaction network was constructed using the STRING database (Szklarczyk et al., 2015). AGI codes of all identified candidates-from yeast 2-hybrid, Co-IP and BioGrid-except UBQ3 (At5G03240), which was excluded because of its ubiquitous interactions, were queried in the STRING database, using a threshold score of 0.4. The resulting network was arranged and color-coded using the cytoscape software tool (Shannon et al., 2003).

\section{AUTHOR CONTRIBUTIONS}

SN generated cDNA libraries and yeast 2-hybrid baits, planned and carried out yeast 2-hybrid experiments, generated Arabidopsis lines stably expressing fluorophore-tagged enzymes, generated lines carrying multiple T-DNA insertions, analyzed glucosinolate data, carried out statistical analysis and confocal microscopy and drafted the manuscript. DV planned, carried out and analyzed Co-IP experiments, carried out GO-term and network analysis and analyzed protein properties. IS carried out and analyzed Co-IP experiments. MBa cloned plasmids and carried out transient expression in Nicotiana benthamiana. KB carried out GO-term analysis and analyzed published transcript abundances. $\mathrm{MBu}$ generated and analyzed glucosinolate data and contributed to statistics, experimental and project design. $\mathrm{BH}$ was involved in experiment planning. All authors commented on the manuscript.

\section{ACKNOWLEDGMENTS}

Financial support was provided by the Danish National Research Foundation DNRF grant $99(\mathrm{SN}, \mathrm{DV}, \mathrm{MBu}$, and $\mathrm{BH})$, the 
Danish Council for Independent Research -FNU 0602-01542B $(\mathrm{MBu}$ and $\mathrm{BH})$ and the Swiss National Science Foundation SNF grant 31003A_162859 awarded to KB and ETH Zurich (JS and $\mathrm{KB})$. Imaging data were collected at the Center for Advanced Bioimaging (CAB) Denmark, University of Copenhagen. The authors would like to thank exchange student Emile Rage for practical help carrying out the untargeted yeast 2-hybrid screens. Project students Jesper Grünberg, Katja Billekop Olsen, and

\section{REFERENCES}

Andersen, T. G., Nintemann, S. J., Marek, M., Halkier, B. A., Schulz, A., and Burow, M. (2016). Improving analytical methods for protein-protein interaction through implementation of chemically inducible dimerization. Sci. Rep. 6:27766. doi: 10.1038/srep27766

Arabidopsis Interactome Mapping Consortium (2011). Evidence for network evolution in an Arabidopsis interactome map. Science 333, 601-607. doi: $10.1126 /$ science. 1203877

Baerenfaller, K., Grossmann, J., Grobei, M. A., Hull, R., Hirsch-Hoffmann, M., Yalovsky, S., et al. (2008). Genome-scale proteomics reveals Arabidopsis thaliana gene models and proteome dynamics. Science 320, 938-941. doi: $10.1126 /$ science. 1157956

Baerenfaller, K., Hirsch-Hoffmann, M., Svozil, J., Hull, R., Russenberger, D., Bischof, S., et al. (2011). pep2pro: a new tool for comprehensive proteome data analysis to reveal information about organ-specific proteomes in Arabidopsis thaliana. Integr Biol 3:225. doi: 10.1039/c0ib00078g

Bak, S., and Feyereisen, R. (2001). The involvement of Two P450 enzymes, CYP83B1 and CYP83A1, in auxin homeostasis and glucosinolate biosynthesis. Plant Physiol. 127, 108-118. doi: 10.1104/pp.127.1.108

Bassard, J.-E., and Halkier, B. A. (2017). How to prove the existence of metabolons? Phytochem. Rev. 1-17. doi: 10.1007/s11101-017-9509-1

Bassard, J.-E., Richert, L., Geerinck, J., Renault, H., Duval, F., Ullmann, P., et al. (2012). Protein-protein and protein-membrane associations in the lignin pathway. Plant Cell 24, 4465-4482. doi: 10.1105/tpc.112.102566

Bateman, A., Martin, M. J., O’Donovan, C., Magrane, M., Apweiler, R., Alpi, E., et al. (2015). UniProt: a hub for protein information. Nucleic Acids Res. 43, D204-D212. doi: 10.1093/nar/gku989

Bednarek, P. (2012). Chemical warfare or modulators of defence responses - the function of secondary metabolites in plant immunity. Curr. Opin. Plant Biol. 15, 407-414. doi: 10.1016/j.pbi.2012.03.002

Bednarek, P., Pislewska-Bednarek, M., Svatos, A., Schneider, B., Doubsky, J., Mansurova, M., et al. (2009). A glucosinolate metabolism pathway in living plant cells mediates broad-spectrum antifungal defense. Science 323, 101-106. doi: 10.1126/science.1163732

Boutet, E., Lieberherr, D., Tognolli, M., Schneider, M., Bansal, P., Bridge, A. J., et al. (2016). Uniprotkb/swiss-prot, the manually annotated section of the uniprot knowledgebase: how to use the entry view. Methods Mol. Biol. 1374, 23-54. doi: 10.1007/978-1-4939-3167-5_2

Brückner, A., Polge, C., Lentze, N., Auerbach, D., and Schlattner, U. (2009). Yeast two-hybrid, a powerful tool for systems biology. Int. J. Mol. Sci. 10, 2763-2788. doi: $10.3390 /$ ijms 10062763

Burow, M. (2016). Chapter two - complex environments interact with plant development to shape glucosinolate profiles. Adv. Bot. Res. 80, 15-30. doi: 10.1016/bs.abr.2016.06.001

Chan, E. K. F., Rowe, H. C., Corwin, J. A., Joseph, B., and Kliebenstein, D. J. (2011). Combining genome-wide association mapping and transcriptional networks to identify novel genes controlling glucosinolates in Arabidopsis thaliana. PLoS Biol. 9:e1001125. doi: 10.1371/journal.pbio.1001125

Chatr-Aryamontri, A., Breitkreutz, B. J., Oughtred, R., Boucher, L., Heinicke, S., Chen, D., et al. (2015). The BioGRID interaction database: 2015 update. Nucleic Acids Res. 43, D470-D478. doi: 10.1093/nar/gku1204

Clay, N. K., Adio, A. M., Denoux, C., Jander, G., and Ausubel, F. M. (2009). Glucosinolate metabolites required for an Arabidopsis innate immune response. Science 323, 95-101. doi: 10.1126/science.1164627
Katrine Wegener Tams are thanked for their contribution to generating yeast 2 -hybrid constructs and initial drop tests.

\section{SUPPLEMENTARY MATERIAL}

The Supplementary Material for this article can be found online at: https://www.frontiersin.org/articles/10.3389/fpls.2017. 02028/full\#supplementary-material
Clough, S. J., and Bent, A. F. (1998). Floral dip: a simplified method forAgrobacterium-mediated transformation of Arabidopsis thaliana. Plant J. 16, 735-743. doi: 10.1046/j.1365-313x.1998.00343.x

Crocoll, C., Halkier, B. A., and Burow, M. (2016). Analysis and Quantification of Glucosinolates. Curr. Protoc. Plant Biol. 1, 385-409. doi: 10.1002/cppb.20027

Dastmalchi, M., Bernards, M. A., and Dhaubhadel, S. (2016). Twin anchors of the soybean isoflavonoid metabolon: evidence for tethering of the complex to the endoplasmic reticulum by IFS and C4H. Plant J. 85, 689-706. doi: 10.1111/tpj.13137

French, J. B., Jones, S. A., Deng, H., Pedley, A. M., Kim, D., Chan, C. Y., et al. (2016). Spatial colocalization and functional link of purinosomes with mitochondria. Science 351, 733-737. doi: 10.1126/science.aac6054

Frerigmann, H., and Gigolashvili, T. (2014). MYB34, MYB51, and MYB122 distinctly regulate indolic glucosinolate biosynthesis in Arabidopsis thaliana. Mol. Plant 7, 814-828. doi: 10.1093/mp/ssu004

Fuchs, R., Kopischke, M., Klapprodt, C., Hause, G., Meyer, A. J., Schwarzländer, M., et al. (2016). Immobilized Subpopulations of Leaf Epidermal Mitochondria Mediate PENETRATION2-Dependent Pathogen Entry Control in Arabidopsis. Plant Cell 28, 130-145. doi: 10.1105/tpc.15.00887

Geu-Flores, F., Nour-Eldin, H. H., Nielsen, M. T., and Halkier, B. A. (2007). USER fusion: a rapid and efficient method for simultaneous fusion and cloning of multiple PCR products. Nucleic Acids Res. 35:e55. doi: 10.1093/nar/gkm106

Gietz, R. D., and Schiestl, R. H. (2007). Frozen competent yeast cells that can be transformed with high efficiency using the LiAc/SS carrier DNA/PEG method. Nat. Protoc. 2, 1-4. doi: 10.1038/nprot.2007.17

Gigolashvili, T., Berger, B., and Flügge, U.-I. (2008). Specific and coordinated control of indolic and aliphatic glucosinolate biosynthesis by R2R3-MYB transcription factors in Arabidopsis thaliana. Phytochem. Rev. 8, 3-13. doi: 10.1007/s11101-008-9112-6

Goedhart, J., von Stetten, D., Noirclerc-Savoye, M., Lelimousin, M., Joosen, L., Hink, M. A., et al. (2012). Structure-guided evolution of cyan fluorescent proteins towards a quantum yield of 93\%. Nat. Commun. 3:751. doi: $10.1038 /$ ncomms 1738

Griffiths, J., Murase, K., Rieu, I., Zentella, R., Zhang, Z.-L., Powers, S. J., et al. (2006). Genetic characterization and functional analysis of the GID1 gibberellin receptors in Arabidopsis. Plant Cell 18, 3399-3414. doi: 10.1105/tpc.106.047415

Gupta, R., Wang, Y., Agrawal, G. K., Rakwal, R., Jo, I. H., Bang, K. H., et al. (2015). Time to dig deep into the plant proteome: a hunt for low-abundance proteins. Front. Plant Sci. 6:22. doi: 10.3389/fpls.2015.00022

Halkier, B. A., and Gershenzon, J. (2006). Biology and biochemistry of glucosinolates. Annu. Rev. Plant Biol. 57, 303-333. doi: 10.1146/annurev.arplant.57.032905.105228

Hansen, C. H., Du, L., Naur, P., Olsen, C. E., Axelsen, K. B., Hick, A. J., et al. (2001). CYP83b1 is the oxime-metabolizing enzyme in the glucosinolate pathway in Arabidopsis. J. Biol. Chem. 276, 24790-24796. doi: 10.1074/jbc. M102637200

Heard, W., Sklenár, J., Tomé, D. F. A., Robatzek, S., and Jones, A. M. E. (2015). Identification of regulatory and cargo proteins of endosomal and secretory pathways in Arabidopsis thaliana by proteomic dissection. Mol. Cell. Proteomics 14, 1796-1813. doi: 10.1074/mcp.M115.050286

Hirsch-Hoffmann, M., Gruissem, W., and Baerenfaller, K. (2012). pep2pro: the high-throughput proteomics data processing, analysis, and visualization tool. Front. Plant Sci. 3:123. doi: 10.3389/fpls.2012.00123

Hooper, C. M., Tanz, S. K., Castleden, I. R., Vacher, M. A., Small, I. D., and Millar, A. H. (2014). SUBAcon: a consensus algorithm for unifying the subcellular 
localization data of the Arabidopsis proteome. Bioinformatics 30, 3356-3364. doi: 10.1093/bioinformatics/btu550

Ihara-Ohori, Y., Nagano, M., Muto, S., Uchimiya, H., and Kawai-Yamada, M. (2007). Cell death suppressor Arabidopsis bax inhibitor-1 is associated with calmodulin binding and ion homeostasis. Plant Physiol. 143, 650-660. doi: $10.1104 / \mathrm{pp} .106 .090878$

Jensen, L. M., Jepsen, H. S. K., Halkier, B. A., Kliebenstein, D. J., and Burow, M. (2015). Natural variation in cross-talk between glucosinolates and onset of flowering in Arabidopsis. Front. Plant Sci. 6:697. doi: 10.3389/fpls.2015.00697

Johansson, O. N., Fantozzi, E., Fahlberg, P., Nilsson, A. K., Buhot, N., Tör, M., et al. (2014). Role of the penetration-resistance genes PEN1, PEN2 and PEN3 in the hypersensitive response and race-specific resistance in Arabidopsis thaliana. Plant J. 79, 466-476. doi: 10.1111/tpj.12571

Jones, A. M., Xuan, Y., Xu, M., Wang, R.-S., Ho, C.-H., Lalonde, S., et al. (2014). Border control-a membrane-linked interactome of Arabidopsis. Science 344, 711-716. doi: 10.1126/science. 1251358

Jørgensen, K., Rasmussen, A. V., Morant, M., Nielsen, A. H., Bjarnholt, N., Zagrobelny, M., et al. (2005). Metabolon formation and metabolic channeling in the biosynthesis of plant natural products. Curr. Opin. Plant Biol. 8, 280-291. doi: 10.1016/j.pbi.2005.03.014

Kawai-Yamada, M., Hori, Z., Ogawa, T., Ihara-Ohori, Y., Tamura, K., Nagano, M., et al. (2009). Loss of calmodulin binding to Bax inhibitor1 affects Pseudomonas-mediated hypersensitive response-associated cell death in Arabidopsis thaliana. J. Biol. Chem. 284, 27998-28003. doi: 10.1074/jbc.M109.037234

Kim, H., O’Connell, R., Maekawa-Yoshikawa, M., Uemura, T., Neumann, U., and Schulze-Lefert, P. (2014). The powdery mildew resistance protein RPW8.2 is carried on VAMP721/722 vesicles to the extrahaustorial membrane of haustorial complexes. Plant J. 79, 835-847. doi: 10.1111/tpj.12591

Kopycki, J., Wieduwild, E., Kohlschmidt, J., Brandt, W., Stepanova, A. N., Alonso, J. M., et al. (2013). Kinetic analysis of Arabidopsis glucosyltransferase UGT74B1 illustrates a general mechanism by which enzymes can escape product inhibition. Biochem. J. 450, 37-46. doi: 10.1042/BJ20121403

Kyte, J., and Doolittle, R. F. (1982). A simple method for displaying the hydropathic character of a protein. J. Mol. Biol. 157, 105-132. doi: 10.1016/0022-2836(82)90515-0

Lalonde, S., Sero, A., Pratelli, R., Pilot, G., Chen, J., Sardi, M. I., et al. (2010). A membrane protein/signaling protein interaction network for Arabidopsis version AMPv2. Front. Physiol. 1:24. doi: 10.3389/fphys.2010.00024

Lamesch, P., Berardini, T. Z., Li, D., Swarbreck, D., Wilks, C., Sasidharan, R., et al. (2012). The Arabidopsis Information Resource (TAIR): improved gene annotation and new tools. Nucleic Acids Res. 40: D1202-D1210. doi: 10.1093/nar/gkr1090

Laursen, T., Borch, J., Knudsen, C., Bavishi, K., Torta, F., Martens, H. J., et al. (2016). Characterization of a dynamic metabolon producing the defense compound dhurrin in sorghum. Science 354, 890-893. doi: 10.1126/science.aag2347

Laursen, T., Møller, B. L., and Bassard, J.-E. (2015). Plasticity of specialized metabolism as mediated by dynamic metabolons. Trends Plant Sci. 20, 20-32. doi: 10.1016/j.tplants.2014.11.002

Li, B., Gaudinier, A., Tang, M., Taylor-Teeples, M., Nham, N. T., Ghaffari, C., et al. (2014). Promoter-based integration in plant defense regulation. Plant Physiol. 166, 1803-1820. doi: 10.1104/pp.114.248716

Liang, H., Yao, N., Song, J. T., Luo, S., Lu, H., and Greenberg, J. T. (2003). Ceramides modulate programmed cell death in plants. Genes Dev. 17, 2636-2641. doi: 10.1101/gad.1140503

Manzano, C., Abraham, Z., López-Torrejón, G., and Del Pozo, J. C. (2008). Identification of ubiquitinated proteins in Arabidopsis. Plant Mol. Biol. 68, 145-158. doi: 10.1007/s11103-008-9358-9

Matsumura, H., Nirasawa, S., Kiba, A., Urasaki, N., Saitoh, H., Ito, M., et al. (2003). Overexpression of Bax inhibitor suppresses the fungal elicitorinduced cell death in rice (Oryza sativa L.) cells. Plant J. 33, 425-434. doi: 10.1046/j.1365-313X.2003.01639.x

Mehrshahi, P., Johnny, C., and DellaPenna, D. (2014). Redefining the metabolic continuity of chloroplasts and ER. Trends Plant Sci. 19, 501-507. doi: 10.1016/j.tplants.2014.02.013

Mehrshahi, P., Stefano, G., Andaloro, J. M., Brandizzi, F., Froehlich, J. E., and DellaPenna, D. (2013). Transorganellar complementation redefines the biochemical continuity of endoplasmic reticulum and chloroplasts. Proc. Natl. Acad. Sci. U.S.A. 110, 12126-12131. doi: 10.1073/pnas.1306331110

Mi, H., Huang, X., Muruganujan, A., Tang, H., Mills, C., Kang, D., et al. (2017). PANTHER version 11: expanded annotation data from Gene Ontology and Reactome pathways, and data analysis tool enhancements. Nucleic Acids Res. 45, D183-D189. doi: 10.1093/nar/gkw1138

Middleton, A. M., Ubeda-Tomas, S., Griffiths, J., Holman, T., Hedden, P., Thomas, S. G., et al. (2012). Mathematical modeling elucidates the role of transcriptional feedback in gibberellin signaling. Proc. Natl. Acad. Sci. U.S.A. 109, 7571-7576. doi: $10.1073 /$ pnas.1113666109

Mikkelsen, M. D., Naur, P., and Halkier, B. A. (2004). Arabidopsis mutants in the C-S lyase of glucosinolate biosynthesis establish a critical role for indole-3-acetaldoxime in auxin homeostasis. Plant J. 37, 770-777. doi: 10.1111/j.1365-313X.2004.02002.x

Möckli, N., Deplazes, A., Hassa, P. O., Zhang, Z., Peter, M., Hottiger, M. O., et al. (2007). Yeast split-ubiquitin-based cytosolic screening system to detect interactions between transcriptionally active proteins. BioTechniques 42 , 725-730. doi: 10.2144/000112455

Nagai, T., Ibata, K., Park, E. S., Kubota, M., Mikoshiba, K., and Miyawaki, A. (2002). A variant of yellow fluorescent protein with fast and efficient maturation for cell-biological applications. Nat. Biotechnol. 20, 87-90. doi: 10.1038/nbt0102-87

Nagano, M., Ihara-Ohori, Y., Imai, H., Inada, N., Fujimoto, M., Tsutsumi, N., et al. (2009). Functional association of cell death suppressor, Arabidopsis Bax inhibitor-1, with fatty acid 2-hydroxylation through cytochrome b5. Plant J. 58, 122-134. doi: 10.1111/j.1365-313X.2008.03765.x

Naur, P., Petersen, B. L., Mikkelsen, M. D., Bak, S., Rasmussen, H., Olsen, C. E., et al. (2003). CYP83A1 and CYP83B1, two nonredundant cytochrome P450 enzymes metabolizing oximes in the biosynthesis of glucosinolates in Arabidopsis. Plant Physiol. 133, 63-72. doi: 10.1104/pp.102. 019240

Nour-Eldin, H. H., Geu-Flores, F., and Halkier, B. A. (2010). USER cloning and USER fusion: the ideal cloning techniques for small and big laboratories. Methods Mol. Biol. 643, 185-200. doi: 10.1007/978-1-60761-723-5_13

Nour-Eldin, H. H., Hansen, B. G., Nørholm, M. H. H., Jensen, J. K., and Halkier, B. A. (2006). Advancing uracil-excision based cloning towards an ideal technique for cloning PCR fragments. Nucleic Acids Res. 34: e122. doi: 10.1093/nar/gkl635

Parisy, V., Poinssot, B., Owsianowski, L., Buchala, A., Glazebrook, J., and Mauch, F. (2007). Identification of PAD2 as a gamma-glutamylcysteine synthetase highlights the importance of glutathione in disease resistance of Arabidopsis. Plant J. 49, 159-172. doi: 10.1111/j.1365-313X.2006.02938.x

Perdigao, N., Heinrich, J., Stolte, C., Sabir, K. S., Buckley, M. J., Tabor, B., et al. (2015). Unexpected features of the dark proteome. Proc. Natl. Acad. Sci. U.S.A. 112, 15898-15903. doi: 10.1073/pnas.1508380112

Petersen, N. H. T., Joensen, J., McKinney, L. V., Brodersen, P., Petersen, M., Hofius, D., et al. (2009). Identification of proteins interacting with Arabidopsis ACD11. J. Plant Physiol. 166, 661-666. doi: 10.1016/j.jplph.2008.08.003

R Core Team (2015). R: A Language and Environment for Statistical Computing. Vienna: R Foundation for Statistical Computing. Avaliable online at: http:// www.R-project.org/

Ralston, L., and Yu, O. (2006). Metabolons involving plant cytochrome P450s. Phytochem. Rev. 5, 459-472. doi: 10.1007/s11101-006-9014-4

Rutter, B., and Innes, R. W. (2016). Extracellular vesicles isolated from the leaf apoplast carry stress-response proteins. Plant Physiol. 173, 728-741. doi: $10.1104 /$ pp.16.01253

Sainsbury, F., Thuenemann, E. C., and Lomonossoff, G. P. (2009). pEAQ: versatile expression vectors for easy and quick transient expression of heterologous proteins in plants. Plant Biotechnol. J. 7, 682-693. doi: 10.1111/j.1467-7652.2009.00434.x

Schlaeppi, K., Bodenhausen, N., Buchala, A., Mauch, F., and Reymond, P. (2008). The glutathione-deficient mutant pad2-1 accumulates lower amounts of glucosinolates and is more susceptible to the insect herbivore Spodoptera littoralis. Plant J. 55, 774-786. doi: 10.1111/j.1365-313X.2008. 03545.x

Shannon, P., Markiel, A., Ozier, O., Baliga, N. S., Wang, J. T., Ramage, D., et al. (2003). Cytoscape: a software environment for integrated models of biomolecular interaction networks. Genome Res. 13, 2498-2504. doi: $10.1101 /$ gr.1239303 
Shen, J., Zeng, Y., Zhuang, X., Sun, L., Yao, X., Pimpl, P., et al. (2013). Organelle $\mathrm{pH}$ in the arabidopsis endomembrane system. Mol. Plant 6, 1419-1437. doi: $10.1093 / \mathrm{mp} / \mathrm{sst} 079$

Smaczniak, C., Li, N., Boeren, S., America, T., van Dongen, W., Goerdayal, S. S., et al. (2012). Proteomics-based identification of low-abundance signaling and regulatory protein complexes in native plant tissues. Nat. Protoc. 7, 2144-2158. doi: $10.1038 /$ nprot.2012.129

Snider, J., Kittanakom, S., Curak, J., and Stagljar, I. (2010). Split-ubiquitin based membrane yeast two-hybrid (MYTH) system: a powerful tool for identifying protein-protein interactions. J. Vis. Exp. 36:1698. doi: 10.3791/1698

Sønderby, I. E., Geu-Flores, F., and Halkier, B. A. (2010). Biosynthesis of glucosinolates-gene discovery and beyond. Trends Plant Sci. 15, 283-290. doi: 10.1016/j.tplants.2010.02.005

Srere, P. (1985). The metabolon. Trends Biochem. Sci. 10, 109-110. doi: 10.1016/0968-0004(85)90266-X

Stagljar, I., Korostensky, C., Johnsson, N., and te Heesen, S. (1998). A genetic system based on split-ubiquitin for the analysis of interactions between membrane proteins in vivo. Proc. Natl. Acad. Sci. U.S.A. 95, 5187-5192. doi: $10.1073 /$ pnas.95.9.5187

Svozil, J., and Baerenfaller, K. (2017). Chapter twenty-one - a cautionary tale on the inclusion of variable posttranslational modifications in databasedependent searches of mass spectrometry data. Methods Enzymol. 586, 433-452. doi: 10.1016/bs.mie.2016.11.007

Szklarczyk, D., Franceschini, A., Wyder, S., Forslund, K., Heller, D., HuertaCepas, J., et al. (2015). STRING v10: Protein-protein interaction networks, integrated over the tree of life. Nucleic Acids Res. 43, D447-D452. doi: $10.1093 /$ nar/gku1003

Vizcaino, J. A., Cote, R. G., Csordas, A., Dianes, J. A., Fabregat, A., Foster, J. M., et al. (2013). The Proteomics Identifications (PRIDE) database and associated tools: status in 2013. Nucleic Acids Res. 41, D1063-D1069. doi: 10.1093/nar/gks1262

von Mering, C., Jensen, L. J., Snel, B., Hooper, S. D., Krupp, M., Foglierini, M., et al. (2005). STRING: known and predicted protein-protein associations, integrated and transferred across organisms. Nucleic Acids Res. 33, D433-D437. doi: $10.1093 /$ nar/gki005

von Mering, C., Krause, R., Snel, B., Cornell, M., Oliver, S. G., Fields, S., et al. (2002). Comparative assessment of large-scale data sets of protein-protein interactions. Nature 417, 399-403. doi: 10.1038/ nature 750

Waterhouse, A. M., Procter, J. B., Martin, D. M. A., Clamp, M., and Barton, G. J. (2009). Jalview Version 2-a multiple sequence alignment editor and analysis workbench. Bioinformatics 25, 1189-1191. doi: 10.1093/bioinformatics/ btp033

Weis, C., Hildebrandt, U., Hoffmann, T., Hemetsberger, C., Pfeilmeier, S., König, C., et al. (2014). CYP83A1 is required for metabolic compatibility of Arabidopsis with the adapted powdery mildew fungus Erysiphe cruciferarum. New Phytol. 202, 1310-1319. doi: 10.1111/nph.12759

Weis, C., Pfeilmeier, S., Glawischnig, E., Isono, E., Pachl, F., Hahne, H., et al. (2013). Co-immunoprecipitation-based identification of putative BAX INHIBITOR1 -interacting proteins involved in cell death regulation and plant-powdery mildew interactions. Mol. Plant Pathol. 14, 791-802. doi: 10.1111/mpp. 12050

Winter, D., Vinegar, B., Nahal, H., Ammar, R., Wilson, G. V., and Provart, N. J. (2007). An "Electronic Fluorescent Pictograph" browser for exploring and analyzing large-scale biological data sets. PLoS ONE 2:e718. doi: 10.1371/journal.pone.0000718

Wu, L., Chen, H., Curtis, C., and Fu, Z. Q. (2014). Go in for the kill. Virulence 5, 710-721. doi: 10.4161/viru.29755

Xu, D., Hanschen, F. S., Witzel, K., Nintemann, S. J., Nour-Eldin, H. H., Schreiner, M., et al. (2016). Rhizosecretion of stele-synthesized glucosinolates and their catabolites requires GTR-mediated import in Arabidopsis. J. Exp. Bot. 68, 3205-3214. doi: 10.1093/jxb/erw355

Yang, Z.-T., Wang, M.-J., Sun, L., Lu, S.-J., Bi, D.-L., Sun, L., et al. (2014). The membrane-associated transcription factor NAC089 controls ERstress-induced programmed cell death in plants. PLoS Genet. 10:e1004243. doi: 10.1371/journal.pgen.1004243

Yun, H. S., Kwaaitaal, M., Kato, N., Yi, C., Park, S., Sato, M. H., et al. (2013). Requirement of vesicle-associated membrane protein 721 and 722 for sustained growth during immune responses in Arabidopsis. Mol. Cells 35, 481-488. doi: $10.1007 / \mathrm{s} 10059-013-2130-2$

Conflict of Interest Statement: The authors declare that the research was conducted in the absence of any commercial or financial relationships that could be construed as a potential conflict of interest.

Copyright (C) 2017 Nintemann, Vik, Svozil, Bak, Baerenfaller, Burow and Halkier. This is an open-access article distributed under the terms of the Creative Commons Attribution License (CC BY). The use, distribution or reproduction in other forums is permitted, provided the original author(s) or licensor are credited and that the original publication in this journal is cited, in accordance with accepted academic practice. No use, distribution or reproduction is permitted which does not comply with these terms. 